\title{
Polymorphism in Tmem132d regulates expression and anxiety-related behavior through binding of RNA polymerase II complex
}

Roshan R. Naik ${ }^{1,2,5}$, Sergey V. Sotnikov ${ }^{1,3}$, Rebekka P. Diepold ${ }^{1}$, Stella lurato ${ }^{1}$, Patrick O. Markt ${ }^{1}$, Andrea Bultmann ${ }^{1}$, Nadine Brehm¹, Tobias Mattheus ${ }^{4}$, Beat Lutz ${ }^{4}$, Angelika Erhardt', Elisabeth B. Binder ${ }^{1}$, Ulrike Schmidt ${ }^{1}$,

Florian Holsboer ${ }^{1}$, Rainer Landgraf ${ }^{1}$ and Ludwig Czibere ${ }^{1,6}$

\begin{abstract}
TMEM132D is a candidate gene, where risk genotypes have been associated with anxiety severity along with higher mRNA expression in the frontal cortex of panic disorder patients. Concurrently, in a high (HAB) and low (LAB) trait anxiety mouse model, Tmem132d was found to show increased expression in the anterior cingulate cortex (aCC) of $H A B$ as compared to $L A B$ mice. To understand the molecular underpinnings underlying the differential expression, we sequenced the gene and found two single-nucleotide polymorphisms (SNPs) in the promoter differing between both lines which could explain the observed mRNA expression profiles using gene reporter assays. In addition, there was no difference in basal DNA methylation in the CpG Island that encompasses the HAB vs. LAB Tmem132d promoter region. Furthermore, we found significantly higher binding of RNA polymerase II (POLR2A) to the proximal HAB-specific SNP (rs233264624) than the corresponding LAB locus in an oligonucleotide pull-down assay, suggesting increased transcription. Virus mediated overexpression of Tmem132d in the aCC of C57BL/6 J mice could confirm its role in mediating an anxiogenic phenotype. To model gene-environmental interactions, HAB mice exposed to enriched environment (HAB-EE) responded with decreased anxiety levels but, had enhanced Tmem132d mRNA expression as compared to standard-housed HAB (HAB-SH) mice. While $L A B$ mice subjected to unpredictable chronic mild stress (LAB-UCMS) exhibited higher anxiety levels and had lower mRNA expression compared to standard-housed LAB (LABSH) mice. Chromatin immunoprecipitation revealed significantly higher binding of POLR2A to rs233264624 in HAB-EE, while LAB-UCMS had lower POLR2A binding at this locus, thus explaining the enhanced or attenuated expression of Tmem132d compared to their respective SH controls. To further investigate gene-environment interactions, DNA methylation was assessed using Illumina $450 \mathrm{~K}$ BeadChip in 74 panic disorder patients. Significant methylation differences were observed in two CpGs (cg26322591 and cg03283235) located in TMEM132D depending on the number of positive life events supporting the results of an influence of positive environmental cues on regulation of Tmem132d expression in mice.
\end{abstract}

Correspondence: Roshan R Naik (rosnaik@gmail.com)

${ }^{1}$ Max Planck Institute of Psychiatry, 80804 Munich, Germany

${ }^{2}$ Department of Behavioral and Molecular Neurobiology, University of

Regensburg, Regensburg, Germany

Full list of author information is available at the end of the article

\section{Introduction}

Anxiety is a state of emotional anticipation to uncertain aversive cues, which helps the organism to adapt to its environment by promoting vigilance and facilitating avoidance behavior ${ }^{1}$. In its normal form, anxiety is a common part of human emotional experience. However, in its pathological form, excessive and inappropriate 
feelings of anxiety can severely disrupt routine functions leading to anxiety disorders. State anxiety is an immediate unpleasant emotional response to a threatening stimulus, while trait anxiety is the existence of more stable and long-term individual response patterns to anxious stimuli. Anxiety disorders are among the most prevalent class of mental disorders and an estimated $20 \%$ of the population are afflicted with it at some point in their lifetime ${ }^{2}$. Both genetic and environmental factors have been shown to be important in mediating anxiety-related behavior. Frequency analysis of anxiety disorders in monozygotic and dizygotic twins reveals that $\sim 30-40 \%$ of the variance in occurrence seen between individuals can be attributed to genetic variation, while the remaining $60-70 \%$ can be attributed to differences in environment ${ }^{3,4}$. Thus. there is a cross-talk between environmental influences and genetic susceptibility factors that interact to mediate anxiety-related behavior. The study of these interactions is important because they can induce plastic, but persistent structural and functional changes in the brain that underlie susceptibility to anxiety and can explain the inter-individual variability in perception of anxiety. Environment enrichment (EE), includes a combination of inanimate and social stimulation as part of housing conditions. It enhances sensory, motor and cognitive abilities in laboratory animals in comparison to their counterparts housed under standard conditions ${ }^{5,6}$. Previous studies have shown that EE causes a decrease in anxiety in mice and is accompanied by lower Crhr1 mRNA expression in the basolateral amygdala (BLA), an important gene and an anatomical site involved in mediating anxiety-like behavior $^{7,8}$. On the other hand, stress is a well-known etiological factor and thus unpredictable chronic mild stress (UCMS) has been utilized to model anxiety- and depression-like behavior ${ }^{9}$ and was found to cause increase in Crhr1 mRNA expression in the BLA of chronically stressed mice ${ }^{8}$. Functional neuroimaging studies in humans have implicated altered activity changes in the anterior cingulate cortex $(\mathrm{aCC})$ or the hippocampus, both closely connected to the amygdala to modulate fear and pathological anxiety responses ${ }^{10-12}$.

The TMEM132D (KIAA1944, MOLT) is a single-pass transmembrane protein with a molecular weight of $\sim 130$ $\mathrm{kDa}$ and although the precise function of the protein is unknown, it is suggested to serve as a cell-surface marker for oligodendrocyte differentiation ${ }^{13}$. RNA-seq transcriptome analysis in mouse cerebral cortex tissue indicates that Tmem132d is expressed in neurons and oligodendrocytes $^{14}$. TMEM132D is more expressed in the cortical regions of the human brain and single-nucleotide polymorphisms (SNPs) (rs11060369, rs7309727) have been associated with panic disorder (PD) and anxiety severity ${ }^{15-17}$. One of these common variants or risk genotype (rs11060369) has been associated with higher
TMEM132D mRNA in the frontal cortex in postmortem brain tissue ${ }^{15}$. Similarly, Tmem132d mRNA is higher expressed in the aCC of high (HAB) compared to low (LAB) anxiety-related behavioral mice ${ }^{15}$. Apart from these common variants, even private coding mutations have been associated with pathological anxiety ${ }^{18}$. A recent study also reported higher anxiety and higher amygdala volume in carriers of the variant (rs11060369) in normal healthy volunteers, suggesting a more generic role of the gene in threat processing ${ }^{19}$. Additionally, a study investigating the quantitative trait loci in mice detected Tmem132d as suggestive locus for fear acquisition ${ }^{20}$. The goal of this study was first to dissect the functional and molecular mechanism underlying differential expression of the Tmem132d gene, and further to investigate gene-environmental interactions in the context of $\mathrm{EE}$ and UCMS in the HAB/LAB mouse model of anxiety-related behavior.

\section{Materials and Methods \\ Animals}

The animal studies were conducted in accordance with the guidelines of the European Union (Council of the European Communities Directive 2010/63/EU) and were approved by the Institutional Animal Care and Use Committee and local authorities (Government of Upper Bavaria). The HAB and LAB animals, which spent $<15 \%$ (HAB) or $>60 \%$ (LAB) of the total test time on one of the open arms of the EPM were bred for over 45 generations in the animal facility of the Max Planck Institute of Psychiatry (MPIP). Suitable numbers of $\mathrm{HAB} \times \mathrm{LAB}$ crosses were made to receive $\mathrm{F} 1$ offspring for behavioral and molecular studies. CD-1 and C57BL/6 J mice were purchased from Charles River (Sulzfeld, Germany). Unlike CD-1 mice, C57BL/6 J is an inbred mouse strain and is the preferred choice for AAV experiment because of its relatively homogenous genetic background and little phenotypic variability. Thus, any difference in phenotype can be traced back to the respective treatment. All animals were housed under optimal conditions, i.e., a temperature of $23 \pm 2^{\circ} \mathrm{C}$, a relative air humidity of $60 \pm 5 \%$ and a 12/12-h light-dark cycle with beginning of the light phase at $8 \mathrm{AM}$ and had access to food pellets (Altromin $\mathrm{GmbH}$, Lage, Germany) and tap water ad libitum. Only male mice were utilized for this study to avoid potential difference in behavioral or molecular effects when female mice are in different stages of estrous cycle. All the behavioral experiments were performed between 08:30 and 11:30 A.M.

\section{Sequencing of Tmem132d}

Genomic DNA was isolated from mouse tail tips (about $6 \mathrm{~mm}$ ) using the NucleoSpin Tissue kit (Macherey-Nagel, Düren, Germany) as per manufacturer's instructions. To 
Table 1 Primers used for sequencing the most relevant fragments of the Tmem132d promoter and exons

\begin{tabular}{|c|c|c|}
\hline Primer set No. & Orientation & Primer sequence $\left(5^{\prime} \rightarrow 3^{\prime}\right)$ \\
\hline 1 & Forward & TGCTGCCAAGCTGTGATAAA \\
\hline 1 & Reverse & TGCGGATAAAAATGGACTGG \\
\hline 2 & Forward & AGCCCAAAACGGACTCTCTT \\
\hline 2 & Reverse & GGACAGTTCAGGAACCCCTA \\
\hline 3 & Forward & TCAGGGACAGGAATTTGAGG \\
\hline 3 & Reverse & TITTAAGCCCCACCCTTCT \\
\hline 4 & Forward & CCAGGAAGGTGGGACCTACT \\
\hline 4 & Reverse & CTGAGGACTGGCTCGTGAAT \\
\hline 5 & Forward & CCAGCCGGAGTCCTCAGA \\
\hline 5 & Reverse & CCACCCACATCCACATCTACT \\
\hline 6 & Forward & AAAGTGAGGCTGTGGGTAGC \\
\hline 6 & Reverse & GTTCTCGTCCCTGTGGTCAT \\
\hline 7 & Forward & CCAACCCATTTGGATTCACT \\
\hline 7 & Reverse & TCCTTCAAAGGTGGACTGCT \\
\hline 8 & Forward & AGGAGGATTCGAGGAAGAGC \\
\hline 8 & Reverse & TCTGCAGACAGTTCCACAGC \\
\hline 9 & Forward & TAAACCTGATTCCCCGTGAG \\
\hline 9 & Reverse & GCCCTGTGTGGGTTCACTAT \\
\hline 10 & Forward & CTTCCTCAAGCTCCTTCTGG \\
\hline 10 & Reverse & GGCTACCCTTGGGATATGGT \\
\hline 11 & Forward & ACAGGTTAATGCCCTGTTGC \\
\hline 11 & Reverse & AGCTTTGCACACTGCACTTC \\
\hline 12 & Forward & GGACTGGGAAAGATGTGGAC \\
\hline 12 & Reverse & GGTCTGGAGAGTGTGGGAAC \\
\hline 13 & Forward & GAGACGTGATAGGCCCATGT \\
\hline 13 & Reverse & CCTITACAGGGGTCGCATA \\
\hline 14 & Forward & TTGTGGATGGTGGTCAGTCC \\
\hline 14 & Reverse & CCATTTTGTCCCCATTITG \\
\hline 15 & Forward & TGTTGCACCAGCGTTGTAC \\
\hline 15 & Reverse & ATCTGGCGTCGCTATTATTAG \\
\hline 16 & Forward & GAAGCACCGTGTGTCTAAGG \\
\hline 16 & Reverse & CCTGGAGCTTGCTGGTCTAC \\
\hline 17 & Forward & ACATTTGGCTTCCTGTGACC \\
\hline 17 & Reverse & CCAGGCATGGGTTATAGCAG \\
\hline 18 & Forward & ATTCCCTGGTCTGTGGACTG \\
\hline 18 & Reverse & GGCAGAGTCTAGGCACTTGG \\
\hline 19 & Forward & AACCACCTGTCATGCCTCTC \\
\hline 19 & Reverse & CCCTGTGTCTCCCATGTATC \\
\hline
\end{tabular}

Table 1 continued

\begin{tabular}{lll}
\hline Primer set No. & Orientation & Primer sequence $\left(\mathbf{5}^{\prime} \boldsymbol{\rightarrow} \mathbf{3}^{\prime} \mathbf{)}\right.$ \\
\hline 20 & Forward & TAATAACATGGGATGGCTGG \\
20 & Reverse & GGGATAGAAACCCCTGATGG \\
21 & Forward & ACCCAATTCTTTCCTCGAC \\
21 & Reverse & TCCACTCTCACTGCTGTTG \\
22 & Forward & TCTCCCTGATGGCTACATCC \\
22 & Reverse & AACAGGTTTCCTGGCCTTC \\
23 & Forward & TGACAGGAGGTCCAAAAAGC \\
23 & Reverse & AGTCCAGACCCCTGTCAATG \\
24 & Forward & TCACTCTCACGACTGGGTTG \\
24 & Reverse & GTGTCCCCGTTATACGTGCT \\
25 & Forward & TCAGACGATGGGTGTCCTTC \\
25 & Reverse & CTGACCCACACATGCTCAAC \\
\hline
\end{tabular}

identify SNPs in Tmem132d, 8,339 bp of the unspliced transcript, $1,132 \mathrm{bp}$ of the promoter and $103 \mathrm{bp}$ of the downstream enhancer region were analyzed. Due to the size of the unspliced transcript $(675,077 \mathrm{bp})$, only exons, fragments of $100-500 \mathrm{bp}$ around exons, furthermore two conserved intronal sequences from intron 3 (717 and 389 bp) and $839 \mathrm{bp}$ from intron 4 were considered for sequencing. The primers (Table 1; Sigma-Aldrich (Taufkirchen, Germany)) for sequencing were designed using NCBI/Primer-BLAST and Tmem132d DNA fragments were amplified using Taq-polymerase (Fermentas, St. LeonRot, Germany) as previously described ${ }^{21}$. Subsequently, PCR products were cleaned up and were utilized for sequencing using Big Dye Terminator kit v3.1 (Applied Biosystems, Foster City, CA, USA), and sequences were resolved by capillary electrophoresis on a 3730 DNA analyzer (Applied Biosystems). Sequence analysis and comparison were performed using FinchTV Ver. 1.2 (Geospiza, Seattle, WA, USA) and BioEdit Ver. 7.0.2 (Tom Hall, Ibis Biosciences, Carlsbad, CA, USA) software programs.

\section{Allele-specific transcription assay (F1 offspring)}

To determine whether differences in the genetic sequence between $\mathrm{HAB}$ and $\mathrm{LAB}$ mice could directly be translated into the previously observed expression differences, crossbred HABxLAB F1 mice were analyzed for the HAB- and LAB-specific alleles' expression. Therefore. qPCR assays were designed to rather amplify the HAB- or the LAB-specific allele based on the SNP identified in exon 3: rs36596918 $(\mathrm{G}(1,747) \mathrm{A})$ or exon 9: rs13478518 (A (3164)G. In addition, CD-1 animals heterozygous for the respective SNP were included in the analysis. One primer of the respective primer pairs were designed to bind to the 
specific allele using 3' end matching and a mismatch base in the penultimate $3^{\prime}$ position of the primer. To correct for assay-specififc differences, results were calculated based on predefined mixtures of HAB and LAB CDNA.

Mice were killed under basal conditions after brief isoflurane anesthesia. Then, the brains were collected and snap-frozen in $\mathrm{N}$-methylbutane and stored at $-80^{\circ} \mathrm{C}$ until further use. Histological staining and atlases ${ }^{22}$ were used to collect samples from the aCC with tissue sample corers having a diameter of $0.8 \mathrm{~mm}$ (Fine Science Tools, Heidelberg, Germany) from $7 \times 200 \mu \mathrm{m}$ slices continuously cooled on dry ice. RNA isolation and qPCR were carried out as decribed before ${ }^{23}$ using primers (Table 2) from Sigma-Aldrich (Taufkirchen, Germany), the Quantifast SYBR Green Kit (Qiagen, Hilden, Germany) on a LightCycler 2.0 (Roche Diagnostics, Mannheim, Germany).

\section{Sequenom assay (CD-1 mice)}

SNPs that were determined by sequencing as described before were validated in $16 \mathrm{HAB}$ vs. $15 \mathrm{LAB}$ mice using a Sequenom MassARRAY iPLEX assay (Sequenom Inc, San Diego, CA, USA). Moreover, 500 phenotyped animals of the second filial generation (F2) of HABxLAB intercross

Table 2 Primers used for the allele-specific expression (ASE) analysis in carriers of both alleles

\begin{tabular}{lll}
\hline Primer & Orientation & Primer sequence $\left(\mathbf{5}^{\prime} \boldsymbol{\rightarrow} \mathbf{3}^{\prime}\right)$ \\
\hline ASE1-e3-HAB & Forward & GCTCTTCAATCTGGGATGTCAG \\
ASE2-e3-LAB & Forward & GCTCTTCAATCTGGGATGTCAA \\
ASE3-cmn-e3 & Reverse & TCGGAGCCAGCAGATTTCTTC \\
ASE4-e9-HAB & Forward & CCTTGGGGCTGGGCTTCATGTG \\
ASE5-e9-LAB & Forward & CCTTGGGGCTGGGCTTCATGTA \\
ASE6-Cmn-e9 & Reverse & TCGGGGCTCCCCCATTCCTG \\
\hline
\end{tabular}

mice ${ }^{24}$ were genotyped, allowing an association study of freely segregating alleles with an anxiety-related phenotype, in addition $80 \mathrm{CD}-1$ mice were included in the analysis.

Primer pairs and single "extension" primers were designed for each SNP in a way that every allele could be genotyped in the same iPLEX-reaction, using a scoring algorithm provided by the manufacturer based on the flanking $100 \mathrm{bp}$ of sequence on both sides of each SNP. Genomic DNA ( $50 \mathrm{ng} / \mu \mathrm{l}$ ) was transferred to 384 well reaction plates. In a primary reaction, the fragments between the corresponding primer pairs were amplified. The remaining dNTPs were dephosphorylated to avoid further binding. In the following iPLEX reaction, the 'extension' primers were extended by only one nucleotide complementary to the respective allele. Like the "extension" primer also the nucleotides had a distinct mass. The scoring software provided by the manufacturer was used to calculate the genotypes based on the mass differences.

\section{In silico analysis for gene regulatory regions}

To identify regions prone to regulation by transcription factors, the PROMO database (http://alggen.lsi.upc.es/ cgi_bin/promo_v3/promo/promoinit.cgi?dirDB =

TF_8.3) 25,26 was utiliized for potential transcription factor-binding sites on the Tmem132d promoter region. After identification of putative transcription factors, their validation was carried out as described in Supplementary Data S1. Genomic coordinates are based on genome builds Mm10 (GRCm38, EnsEMBL release 68) for mice.

\section{Dual luciferase assays}

To examine the role of the identified polymorphisms $\sim 600 \mathrm{bp}$ of the putative promoter region was cloned into pGL3-basic luciferase vectors and transfected into mouse Neuro-2a cells using Exgen 500 in vitro reagent (Thermofisher Scientific, Darmstadt, Germany; Table 3 for

Table 3 Primers used for cloning Tmem132d promoter fragments into the luciferase vector

\begin{tabular}{lll}
\hline Primer & Orientation & Primer sequence $\mathbf{5}^{\prime} \rightarrow \mathbf{3}^{\prime} \mathbf{)}$ \\
\hline RVprimer3 & Forward & CTAGCAAAATAGGCTGTCCC \\
GLprimer2 & Reverse & CTTTATGTTTTGGCGTCTTCCA \\
Tmem132d & Forward & GCAGGTACCCAAGGCTCTGCGGAGCAGTG \\
Tmem132d & Reverse & GCTAGCAATTTCTCTCTCTTCCTCTCTCCC \\
Tmem132dreverse310 A sub & Forward & GTTAGGGTTCCTGAACTGTCCTTGCCTGAAG \\
Tmem132dreverse310 A sub & Reverse & CTTCAGGCAAGGACAGTTCAGGAACCCCTAAC \\
Tmem132dreverse310 del & Forward & GACGGTACCTCAGGGACAGGAATTGAGG \\
Tmem132dreverse519 A sub & Forward & GTGTGAGTCGCCTTAGATACCCTGGAAGG \\
Tmem132dreverse519 A sub & Reverse & CCTTCCAGGGTATCTAAGGCGAACTCACAC \\
\hline
\end{tabular}


primer list). The Tmem132d promoter constructs were co-transfected with a pRK5-Gaussia-KDEL vector to normalize transfection efficiency and a SV40-pGL3 vector was used as a positive control. The cells were lysed $48 \mathrm{~h}$ post-transfection and processed in a dual luciferase assay as described in $^{27}$.

\section{Oligonucleotide pull-down assay}

In order to confirm the functionality of SNP in the Tmem132d promoter region $(-310$ and -519$)$, we performed oligonucleotide pull-down assays to assess binding of POLR2A.

Annealing of oligos was carried out by mixing equal amounts of $5^{\prime}$-biotinylated forward oligonucleotides containing either the $\mathrm{HAB}$ or $\mathrm{LAB}$ sequence variant and respective non-biotinylated strands (Table 4) along with $10 \times$ NEB buffer 2 . The reaction mixture was incubated for $4 \mathrm{~min}$ at $95^{\circ} \mathrm{C}$, followed by $10 \mathrm{~min}$ at $70{ }^{\circ} \mathrm{C}$ and finally left on the benchtop for 4-5 h, which allowed annealing of the oligonucleotides. Then, streptavidin beads (GE Healthcare Europe GmbH, Freiburg, Germany) were washed with ice-cold PBS and incubated overnight with the annealed 5'-biotinylated oligonucleotides.

Nuclear extracts from CD-1 mouse brain tissue were prepared using the NE-PER nuclear and cytoplasmic extraction reagent (Thermofisher Scientific), precleared using streptavidin beads and protein concentration estimated using the Pierce BCA protein assay kit (Thermofisher Scientific). Then, $300 \mu \mathrm{g}$ of nuclear proteins were incubated with the biotinylated double strandedstreptavidin sepharose complex for $4 \mathrm{~h}$ on a rotating shaker, washed with RIPA buffer, heated at $95^{\circ} \mathrm{C}$ in sample buffer and separated on 10\% SDS-PAGE. The separated proteins were blotted on a nitrocellulose membrane, non-specific binding blocked using 5\% milkTBST and incubated overnight with a polyclonal POLR2A antibody (sc-899×, Santa Cruz Biotechnology, Inc. Frankfurt am Main, Germany) at 1:150 dilution in 2.5\% milk in TBST. Subsequently, the blot was washed $3 \times$
TBST for $10 \mathrm{~min}$ each and incubated for $2 \mathrm{~h}$ at room temperature with anti-rabbit HRP-conjugated secondary antibody (1:1000) diluted in $2.5 \%$ milk/TBST. Finally, the blot was washed again with $3 \times$ TBST for 10 min each and proteins were visualized via chemiluminescence (Western Lighting, Perkin Elmer, Frankfurt am Main, Germany). The protein loading control was carried out by subjecting the blot to colloidal silver staining ${ }^{28}$.

\section{Adeno-associated virus (AAV) mediated overexpression of Tmem132d}

To confirm the role of Tmem132d in anxiety-related behavior, its corresponding cDNA was amplified from pcDNA 3.1/V5- m-MOLT (kindly provided by Prof. Tomoko Yonezawa, Okayama University Graduate School of Medicine, Japan) using the primers described in Table 5. The Tmem132d cDNA sequence was sequenced and confirmed to be identical to the LAB Tmem132d cDNA i.e., there were no polymorphisms. Further, we obtained an AAV expression cassette (pAM-CBA-MCSWPRE2-SpA-containing CMV immediate early enhancer/ chicken beta-actin hybrid promoter CBA, multiple cloning site, woodchuck hepatitis virus post-transcriptional regulatory element WPRE2, and SV40 late polyadenylation site). A shorter $0.4 \mathrm{~Kb}$ neuronal CamKII promoter (to replace $1.1 \mathrm{~kb}$ CBA promoter) and an eGFP fluorophore (to confirm viral spread) both derived from Addgene plasmid (\# 27226) ${ }^{29}$ were cloned into the AAV expression cassette. Instead of fluorophore, a $3.3 \mathrm{~kb}$ of Tmem132d cDNA was also cloned into AAV expression cassette and the corresponding plasmid was transfected into Neuro-2a cells to confirm Tmem132d overexpression using qPCR. Production of pseudotyped $\mathrm{rAAV} 1 / 2$ mosaic vectors and determination of genomic titers were performed as described in ref. ${ }^{30}$. Eight-week-old male C57BL/6J mice were anesthetized by inhalation of isoflurane (Baxter Germany GmbH, Unterschleißheim, Germany) and placed on a thermo pad $\left(32^{\circ} \mathrm{C}\right)$ to minimize core body temperature loss. Initially, 2-3 mice were stereotactically

Table 4 Primers used for the oligonucleotide pull-down assay

\begin{tabular}{|c|c|c|}
\hline Primer & Orientation & Primer sequence $\left(5^{\prime} \rightarrow 3^{\prime}\right)$ \\
\hline Tmem132dreverseLABreverse519reverseBiotin & Forward & GTTCGCCTTAGATACCCT \\
\hline Tmem132dreverseLABreverse519 & Reverse & AGGGTATCTAAGGCGAAC \\
\hline Tmem132dreverseLABreverse310reverseBiotin & Forward & GGTTCCTGAACTGTCCTT \\
\hline Tmem132dreverseLABreverse 310 & Reverse & AAGGACAGTTCAGGAACC \\
\hline Tmem132dreverseHABreverse519reverseBiotin & Forward & GTTCGCCTTGGATACCCT \\
\hline Tmem132dreverseHABreverse519 & Reverse & AGGGTATCCAAGGCGAAC \\
\hline Tmem132dreverseHABreverse310reverseBiotin & Forward & GGTTCCTGAGCTGTCCTT \\
\hline Tmem132dreverseHABreverse 310 & Reverse & AAGGACAGCTCAGGAACC \\
\hline
\end{tabular}


Table 5 Primers used Tmem132d mRNA expression studies and cloning of the Tmem132d CDNA into the AAV expression cassette

\begin{tabular}{lll}
\hline Primer & Orientation & Primer sequence $\left(\mathbf{5}^{\prime} \rightarrow \mathbf{3}^{\prime}\right)$ \\
\hline Tmem132dreverseexp & Forward & AGGAGCTGGGCATGACCA \\
Tmem132dreverseexp & Reverse & GAGGTCTGTGATGGTCACCTT \\
Camkllp & Forward & CTAGGGTACCCTTGTGGACTAA \\
& & GTTGTTCACATCC \\
Camkllp & Reverse & CTAGCTCGAGCGGGGATCGGC \\
& & TCTAGAG \\
Tmem132d & Forward & CTAGGAATCGCCACCATGTGC \\
& & CCATCTGAGATGGGGA \\
Tmem132d & Reverse & CTAGGAATTCAAGCTITATAC \\
& & GTGCTCGTGTAACCTCTCCAT \\
\hline
\end{tabular}

injected with a miniscule amount of Nissl's stain bilaterally into the aCC $(+1.5 \mathrm{~mm} \mathrm{AP},+0.2 \mathrm{~mm} \mathrm{ML}, 1.4 \mathrm{~mm}$ DV) from Bregma and then the brain was collected and sectioned to confirm its localization. Another 2-3 mice had AAV-eGFP injected unilaterally (contralateral side internal control with no injection) to confirm viral localization and spread. Subsequently, other mice were randomly assigned using simple randomization to receive either $0.5 \mu \mathrm{l}$ of $1 \times 10^{11}$ AAV-empty or AAV-Tmem $132 d$ vector copies/ml via stereotaxic injection bilaterally into the aCC coordinates as described above. The vector delivery was performed using a microinfusion pump at a rate of $0.5 \mu \mathrm{l} / 10 \mathrm{~min} .2 \mathrm{~min}$ after the injection, the needle was carefully retracted and the scalp sutured. Mice were treated with $100 \mu \mathrm{l}$ Enrofloxacin (Baytril 2.5\%; Bayer Vital $\mathrm{GmbH}$, Leverkusen, Germany) s.c. to prevent inflammation and transferred to their home cage for 3 weeks until behavioral testing for anxiety-related behavior. Moreover, Tmem132d gene expression was quantified in aCC region harvested from AAV-empty vs. AAV-Tmem132d injected mice. AAV-eGFP injected mice were anesthetized with sodium pentobarbital $(200 \mathrm{mg} / \mathrm{kg})$ and transcardially perfused with phosphate buffered saline (PBS), 4\% paraformaldehyde (PFA) and the isolated whole brain was postfixed overnight in $4 \%$ PFA at $4{ }^{\circ} \mathrm{C}$. Subsequently, the brain was rinsed in PBS and immersed in $30 \%$ sucrose (to cryoprotect tissue) until brain sinks to the bottom of the tube. Then the brain was snap frozen with $n$-methyl butane and transferred to $-80^{\circ} \mathrm{C}$ freezer overnight. Eventually, the brain was embedded in OCT medium (VWR GmbH, Darmstadt, Germany) and cryosectioned into $30 \mu \mathrm{m}$ thick coronal section, which was imaged for eGFP with a $4 \times$ objective on a wide-field fluorescent inverted Leica DM16000 microscope.

\section{Behavioral paradigms}

Environmental enrichment (EE): EE paradigm was adapted from $\mathrm{Li}$ et $\mathrm{al}^{31}$ and Arai et $\mathrm{al}^{32 \text {, }}$ and is composed of a combination of group housing ( 3 mice per cage) and biologically relevant stimuli such as wood chips and scaffold, plastic insets and tunnel, which along with a bigger home cage allowed mice to interact with their environment and accomplish highly motivated natural behaviors. The EE paradigm consisted of two 14-day periods, called partial and full EE. The partial EE begins from postnatal day (PND) 15-28, when the pups along with their respective dam were transferred for $6 \mathrm{~h}$ per day into the bigger cages with different manipulanda. At PND28, pups were weaned and sorted in groups of three and moved for complete EE permanently until PND42.

Unpredictable chronic mild stress (UCMS): The UCMS protocol was adapted from Willner et $\mathrm{al}^{33}$ and the timeline was similar to EE. It consisted of alternating mild stressors such as maternal separation, absence of bedding (sawdust), cage tilting $\left(45^{\circ}\right)$, mild foot shock (PND15-28) followed by other stressors such as overcrowding, wet bedding, overnight illumination, restraint stress, white noise from PND 29 to 42 to elicit an anxiogenic and prodepressive phenotype.

The standard HAB and LAB mice were housed under normal conditions without any manipulations (standard housing: $\mathrm{SH}$ ).

\section{Elevated-plus maze (EPM) test}

The EPM test consists of two open arms $(30 \times 5 \mathrm{~cm})$ and two closed arms $(30 \times 5 \times 15 \mathrm{~cm})$ joined by a central zone $(5 \times 5 \mathrm{~cm})$ raised $40 \mathrm{~cm}$ above the floor. Light intensity on open and closed arm were 300 lux and $<10$ Lux, respectively, while 50 lux in the central zone. The entries and percent time spent on open arm was taken as an index of anxiety-related behavior, while entries and time spent in closed arm serves as an indicator of locomotion during the 5 min test between 8:30 to 11:30 A.M.

\section{Light-dark box (LDB) test}

To examine anxiety-related behavior, the LDB test was carried out for $5 \mathrm{~min}$ between 8:30 and 11:30 A.M, which measures percent time spent and distance traveled in the light compartment as well as latency to enter and entries in the light compartment. The LDB test consisted of a dark $(16 \times 27 \times 27 \mathrm{~cm})$ and a light compartment $(32 \times 27 \times 27$ $\mathrm{cm})$ illuminated with $<20$ Lux and 400 Lux, respectively. EthoVision XT 5.0 (Noldus Information technology, Wageningen, the Netherlands) software was utilized to track animal movements throughout the test duration.

qPCR

One cohort of animals were used for RNA extraction, cDNA preparation and real-time PCR from the aCC tissue 
as described above on a LightCycler 2.0 (Roche Diagnostics, Mannheim, Germany) or 7500 Real-time PCR System (Applied Biosystems, CA, USA). Each experiment was performed in duplicates, standard curves were generated and the relative transcript concentrations were calculated using the $2^{(-\Delta \Delta \mathrm{Ct})}$ method $^{34}$. The primerpair is listed in Table 5.

\section{Chromatin immunoprecipitation (ChIP) assay}

To confirm in vivo POLR2A binding and quantification to the Tmem $132 d$ promoter A(-310)G SNP in HAB-SH, LAB-SH, HAB-EE, and LAB-UCMS, we utilized ChIP assay using the ChIP-IT express kit (Active Motif, La Hulpe, Belgium) with certain modifications.

aCC tissue $(>50 \mathrm{mg}$ ) was cross-linked at room temperature for $10 \mathrm{~min}$ in ice-cold $1 \%$ formaldehydephosphate buffered saline (PBS). Then cross-linking was stopped by adding glycine to a final concentration of 0.125 $\mathrm{M}$ and incubated at room temperature for $5 \mathrm{~min}$ and centrifuged to collect the pellet. Then the pellet was resuspended in lysis buffer and sonicated by applying seven $30 \mathrm{~s}$ pulses at high power using standard bioruptor (Diagenode s.a., Liège, Belgium). The sheared chromatin was utilized for immunoprecipitation with POLR2A antibody (sc-899×, Santa Cruz Biotechnology) and protein A/ G plus-agarose (sc-2003, Santa Cruz Biotechnology) along with buffers provided in the ChIP-IT express kit. The immunoprecipitated DNA-protein $\mathrm{A} / \mathrm{G}$ plus agarose complex was subsequently washed, eluted, reverse crosslinked and treated with proteinase $\mathrm{K}$ to obtain the enriched DNA fraction as described in the ChIP-IT express kit. The end point quantitative PCR was carried out using primers flanking the $\mathrm{A}(-310) \mathrm{G}$ SNP region (Table 6) and also mouse positive and negative primers (Active Motif) for POLR2A binding at active and inactive regions.

\section{Data analysis}

Behavioral phenotypic and gene expression differences were analyzed using either the two-tailed Student's $t$ test or one-way ANOVA with post hoc Bonferroni's corrections using GraphPad Prism software version 7.02 (La Jolla, CA) and significance was accepted at $p<0.05$. The results are presented as mean \pm s.e.m. with animal number and replicates indicated in figures or figure legends. Animal sample size was justified by previously published

Table 6 Primers used for qPCR end point analysis of ChIP enriched fractions of POLR2A

\begin{tabular}{lll}
\hline Primer & Orientation & Primer sequence $\left.\mathbf{( 5}^{\prime} \boldsymbol{\rightarrow} \mathbf{3}^{\mathbf{\prime}}\right)$ \\
\hline Tmem132dreverse310 & Forward & ATTTGGCATCCCAGGCTCA \\
Tmem132dreverse310 & Reverse & AGGTGTGGAAAGGCCATCTG \\
\hline
\end{tabular}

data $^{8,35}$ or preliminary experiments. All experimental animals with similar behavioral profile (e.g., HABs for standard-housing or environmental enrichment) or wildtype mice were randomly allocated to experimental groups. The distribution of the data was assumed to be normal; however, this was not formally tested. Whenever possible, the experimenter was blind to experimental and/ or treatment group.

\section{Methylation analysis in patients with anxiety disorders}

PD patients were recruited at the outpatient unit of the Max Planck Institute of Psychiatry (MPIP) in Munich ${ }^{15}$. All patients included in the current analyses $\left(N_{\text {total }}=74, N_{\text {males }}\right.$ $=26, N_{\text {females }}=48$ ) were Caucasian and were not taking any psychotropic medications. Anxiety symptoms were assessed with the Hamilton Anxiety scale (HAMA) ${ }^{36}$ and severity of panic symptoms was assessed with the Panic and Agoraphobia scale $(\mathrm{PAS})^{37}$. Positive Life Events were assessed using a German modified version ${ }^{38}$ of the Social Readjustment Rating Scale ${ }^{39}$. All subjects provided written informed consent and procedures were approved by the Ethics Committee of the Ludwig Maximilians University, Munich, Germany, in accordance with the Declaration of Helsinki.

\section{DNA methylation arrays in the MPIP Study}

Genomic DNA was extracted from peripheral blood using the Gentra Puregene Blood Kit (Qiagen). DNA integrity and concentration was assessed by NanoDrop 2000 Spectrophotometer (Thermo Scientific) and QuantiT Picogreen (Invitrogen). Genomic DNA was bisulfite converted using the Zymo EZ-96 DNA Methylation Kit (Zymo Research) and DNA methylation levels were assessed for $>480,000$ CpG sites using the Illumina HumanMethylation450 BeadChip array. Hybridization and processing was performed according to the instructions of the manufacturer. Quality control of the methylation data included intensity read outs, normalization, cell type composition estimation, $\beta$ - and $\mathrm{M}$-value calculation and was performed using the Bioconductor R package "minfi" (version 1.10.2). X-chromosome, Y-chromosome, and nonspecific binding probes were removed ${ }^{41}$ together with 236 failed probes that were excluded based on a detection pvalue larger than 0.01 in $>50 \%$ of the samples. Probes containing a SNP in the sequence or located close (10 bp from query site) to a SNP, which had a minor allele frequency of $\geq 5 \%$ were also removed. The data were then normalized using functional normalization ${ }^{42}$, an extension of quantile normalization included in the $R$ package "minfi". Batch effects were identified by inspecting the association of the first principal component of the methylation levels with bisulfite conversion plate, well, slide, slide row and array using linear regression and visual inspection of PCA plots using the Bioconductor R package 
shinyMethyl (version 0.99.3). The Empirical Bayes' (EB) method ComBat was used to remove batch effects.

\section{Gene-environment interaction analysis}

To test whether there was an association between TMEM132D methylation levels and the number of positive life events experienced depending on previously described risk alleles for PD in the rs7309727 SNP $\left(N_{\text {TT }}\right.$ $=38, N_{\mathrm{CT}}=36, N_{\mathrm{CC}}=0$ ), a linear regression model was fitted with the batch corrected M-values for each of the 73 CpGs located in the TMEM132D gene. Gender, age and Houseman-calculated blood cell counts ${ }^{42}$ were included as covariates in all the regression models.

\section{POLR2A ChIP-seq of TMEM132D gene}

The RNA Pol II ChIP-seq data have been obtained with K562 chronic myelogenous leukemia ${ }^{43}$ and GM7 $^{44}$ lymphoblastoid cell lines by Michael Snyder lab at Yale University (GEO accession number GSE13008 and GSE30399, respectively). These data are made publicly available by ENCyclopedia Of DNA Elements (ENCODE) analysis on UCSC genome browser (http://genome.ucsc. $\mathrm{edu} /)^{45-47}$, and were utilized to view the POLR2A ChIPseq data for TMEM132D gene on the human feb. 2009 (GRCh37/hg19) Assembly.

\section{Results}

\section{Polymorphisms in the Tmem132d gene}

To understand the differential expression of the Tmem132d mRNA in the aCC of HAB vs. LAB, its putative promoter region up to $1000 \mathrm{bp}$ and coding regions were sequenced. Sequencing Tmem132d, several polymorphic loci could be identified, among them, two SNPs in the promoter of the gene at positions $\mathrm{A}(-519) \mathrm{G}$ and rs233264624 $(\mathrm{A}(-310) \mathrm{G})$. Four further SNPs were identified located in exons, with $\mathrm{C}(470) \mathrm{T}$ and $\mathrm{C}(593) \mathrm{T}$ in the 5' untranslated region and $\mathrm{G}(1,747) \mathrm{A}$ in exon 3 (also known as rs36596918) and $\mathrm{A}(3,164) \mathrm{G}$ in exon 9 (rs13478518) as part of the protein coding sequence. Furthermore, three SNPs and two deletions were discovered in introns, among them $\mathrm{C}(128,616,797) \mathrm{T}$ (rs13478520), A $(128,577,729) \mathrm{T} \quad$ (rs6387514), $\Delta(128,577,906-128,577,903)$ (rs237178791) and $\Delta(128,577,978-128,577,974)$ in the third and $\mathrm{T}(128,344,853) \mathrm{A}$ in the fifth intron (Fig. 1a and Table 7).

\section{Allele-specific transcription of Tmem $132 d$}

The analysis of allele-specific transcription based on $\mathrm{G}(1,747) \mathrm{A}$ (rs36596918) revealed a significantly higher transcription of the HAB-specific allele with both alleles present in the same cell. This held true for HABxLAB F1 offspring and CD-1 mice heterozygous for Tmem132d (" $\mathrm{p}<0.05$ and ${ }^{* * * *} p<0.001$, respectively, Figs. 1b, c).

\section{Genotype-phenotype associations (Sequenom assay)}

In the HAB-LAB-derived F2 mice ${ }^{24}$, all SNPs discovered in $\mathrm{HAB}$ vs. $\mathrm{LAB}$ mice and analyzed in Tmem132d displayed similar distribution patterns, as the genomic locus is not larger than $800 \mathrm{kbp}$, however, some recombination has occurred over two breeding generations. Altogether, 10 recombination events have been seen in 501 mice over two generations, thus resulting in a recombination rate of 5 per generation or 1 /generation and 100 individuals, i.e., a recombination rate of $1 / 100$ within the given locus. Thus, the association of Tmem132d genotypes with anxiety-related behavior, as described ${ }^{15}$ remains significant for all SNPs in Tmem132d $(p<0.01)$. The additional analysis of 80 unrelated CD-1 mice revealed, that rs13478518 (the SNP in exon 9) showed higher variation, as the other SNPs did, with the HAB-specific allele being the minor allele (minor allele frequency-MAF $=0.39$ ). For the other SNPs described for Tmem132d in CD-1 mice, the HAB-specific variations were also the minor alleles, with $\mathrm{MAF}=0.08$. However, due to the small sample size, genotype-phenotype associations did not reveal any significant association in CD-1 mice.

\section{Promoter SNPs are sufficient to explain the higher Tmem132d mRNA expression}

The LAB vs. HAB Tmem $132 d$ promoter carrying the A $(-519) \mathrm{G}$ and $\mathrm{A}(-310) \mathrm{G}$ SNPs, respectively were cloned into pGL3-basic vector and promoter activity was measured using dual luciferase assay. The whole HAB Tmem132d promoter constructs containing guanine nucleotide(s) at the positions $(-519)$ and $(-310)$ exhibited higher luciferase expression $(* * p<0.01$; Fig. 2a) compared to corresponding LAB constructs, suggesting increased promoter activity. These data are in line with the in vivo findings of higher Tmem132d mRNA expression in HAB-SH compared to LAB-SH. To dissect the individual contribution of SNPs, mutation of the guanine nucleotide to adenine at $(-519)$ or $(-310)$ caused a significant decrease in promoter activity $\left({ }^{* * * *} p<0.001\right.$ and $* *<0.01$ ), respectively in comparison to whole HABsequence-based constructs. This indicates that both guanine residues are important for the higher $\mathrm{HAB}$ promoter activity. Meanwhile, deletion of the A nucleotide at position $(-519)$ in the LAB construct did not have any effect on its promoter activity. While, the deletion of the G nucleotide at $(-519)$ in the HAB construct again led to significant decrease in promoter activity $(* * p<0.01$; Fig. 2a), thus proving the importance of the $\mathrm{G}$ nucleotide at $(-519)$ for the observed expression.

The discovery of a $\mathrm{CpG}$ island in the upstream Tmem132d promoter prompted us to do bisulfite sequencing (Table 8). There was no difference in total DNA methylation between HAB/LAB basal Tmem132d promoter regions. Supplementary Data S2 illustrates these 


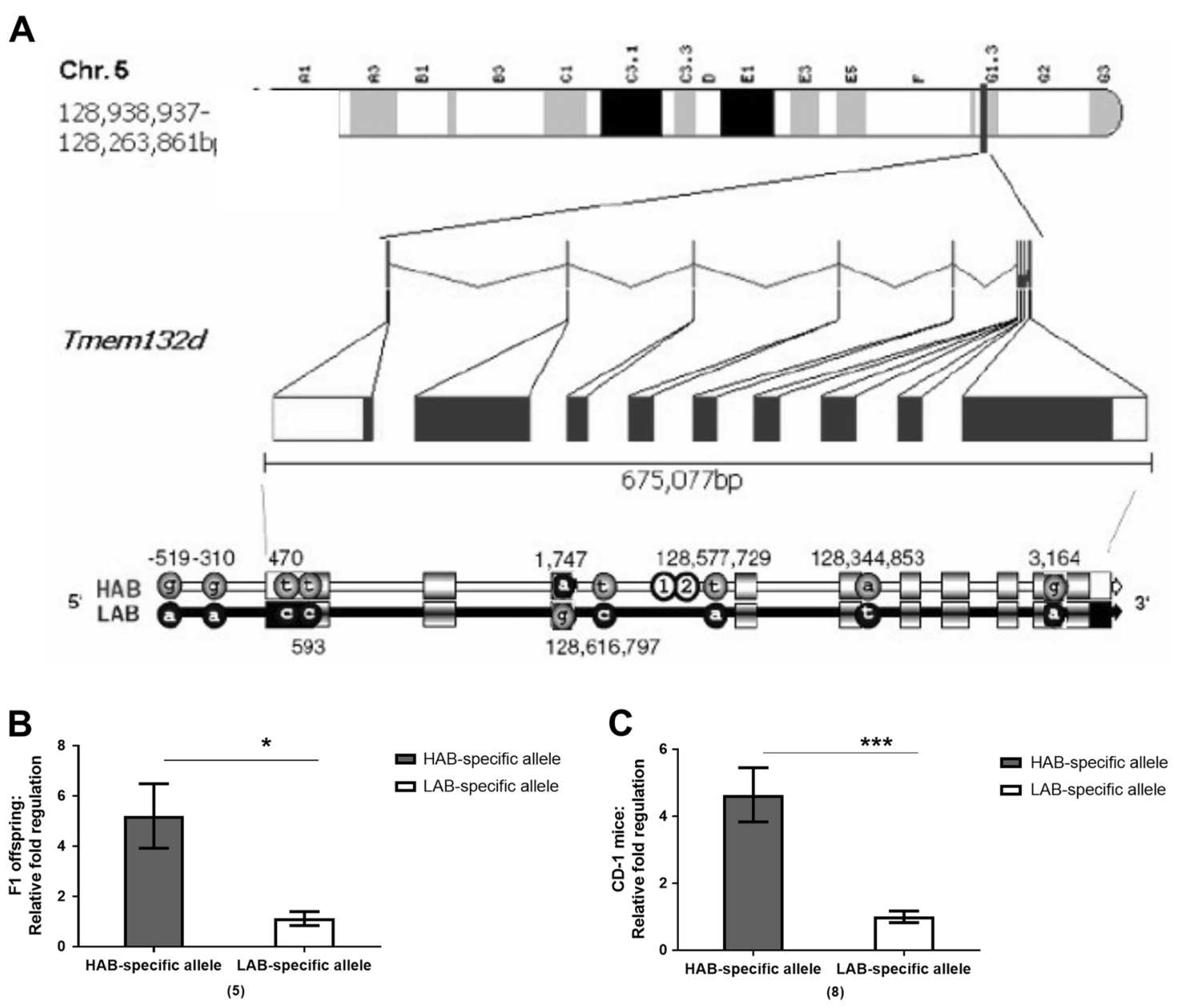

Fig. 1 a Transmembrane protein 132D (Tmem132d) gene sequence of HAB vs. LAB mice. Polymorphic sites are indicated with minus sign for promoter positions in bp, with positions in the coding sequence from transcription start in the spliced mRNA (in bp) or with chromosomal positions for single-nucleotide polymorphisms in introns (in bp). Exons and untranslated regions (UTRs) are indicated by boxes (exons shaded, UTRs completely filled black or white). Unfilled circles with numbers ' 1 ' and ' 2 ' refer to two deletions in HAB mice. For ' 1 ' at position 128,577,978 bp (deletion of CAAA) and ' 2 ' at position 128,577,906 (deletion of ACA). Allele-specific transcription assay in HABXLAB cross-mated F1 b and CD-1 c mice heterozygous for rs36596918. The data are presented as mean \pm SEM and numbers in parentheses indicate the group size; ${ }^{* * *} p<0.0001$; ${ }^{*} p<0.05$

findings. Selective or complete methylation of Tmem132d promoter (Table 9) did not alter its promoter activity (data not shown).

\section{Binding of putative transcription factor at regulatory regions of Tmem132d}

The oligonucleotide pull-down assay indicated that binding of the transcription factor POLR2A occurred at significantly higher levels to the HAB-locus containing the $\mathrm{G}$ nucleotide at position $(-310)$ as compared to corresponding LAB-specific locus $(p<0.05$; Fig. $2 \mathrm{~b})$. The band size was, as expected, approximately $250 \mathrm{kDa}$ in size (Fig. 2b). In addition, there was an unknown band at $\sim 125 \mathrm{kDa}$ of equal intensity in all samples (Fig. 2b).
Higher expression of Tmem132d in the aCC causes increased anxiety-related behavior

Overexpression of the Tmem132d gene using an AAVmediated system in the aCC caused C57BL/6 J mice to spend significantly lower percent time in open arm of EPM and light compartment of LDB test compared to mice injected with empty control AAV $(\mathrm{p}<0.05$; Fig. 3a, $c$, respectively). Furthermore, there was no difference in locomotion between the two groups of mice (Fig. 3b, d). Animals injected with Tmem132d-AAV have sparse tracks in the light compartment compared to those injected with empty-AAV (Fig. 3e, f). We could also confirm higher Tmem132d mRNA in the Tmem132dAAV injected mice relative to empty AAV $(p<0.001$; 
Table 7 Overview of the identified polymorphisms in Tmem132d in HAB vs. LAB mice including the flanking sequences. The HAB polymorphisms are underlined

\begin{tabular}{|c|c|c|}
\hline $\begin{array}{l}\text { Genetic } \\
\text { region }\end{array}$ & & $3^{\prime}$ \\
\hline \multirow[t]{2}{*}{$\begin{array}{l}\text { promoter } \\
\text { region }\end{array}$} & $A(-519) \underline{G}$ & $\begin{array}{l}\text { тाCCTCTCTGAATCCCCACAACATCTGCTTGTTTCCAAGGCTCTGCGGAGCAGTGAGGAGGGCTCCGGCTCGGGACACTAGCATG } \\
\text { GGTATTATGTATCTTGGTGTGAGTTCGCCTT[A/G]GATACCCTGGAAGGCATGAGTGGAGAGCTGTACCTATTAGCTITCAATGA } \\
\text { AATATCTTCCCTAATGCCAATTCCCTAATGCCCAAAGGCCAGCGGGGAGCCAGGATGGAAATCTाT }\end{array}$ \\
\hline & $\begin{array}{l}A(-310) \underline{G} \\
\text { rs233264624 }\end{array}$ & $\begin{array}{l}\text { CGGGGAGCCAGGATGGAAATCTTTCAGGGACAGGAATTTGAGGGTCATTTTGGCATCCCAGGCTCACAATCTAGAGTTCAC } \\
\text { CGGAAACACTTCAGCCTATGTTAGGGGTTCCTGA[A/G]CTGTCCTTGCCTGAAGGCCGCCTATAGTCAGAAGCTTAAGAAGGC } \\
\text { AGGGCAGATGGCCTTTCCACACCTGTTGTGAGCGTGGACATCCACGCTGGTCTCGTGCGCGCTCCTCGGGGCTTGCGGGGT } \\
\text { GTCCATAACCTC }\end{array}$ \\
\hline \multirow[t]{2}{*}{$\begin{array}{l}\text { exon } 15^{\prime} \\
\text { untranslated } \\
\text { region }\end{array}$} & $C(470) \underline{I}$ & $\begin{array}{l}\text { GCAGCCCCGCAGCCGCCCGCGCGCGCTAGCCCACCGCAGCCTCCCAGCCGGAGTCCTCAGAGCGCTCTCCATGCTTGGGGA } \\
\text { CGCAACACCGGAGACCCCCCTTGTGGAGTGAAATCAATTCACGAGCIC/[_AGTCCTCAGCGCAGTCGAAAGACTGAGGTGA } \\
\text { GCCTCGGCCTGCGCTGCGGGACCCCGGAGCCTGAGCGGCCTGGCTATGGACCTGTGGGGACCCGGCACAGGGCGGCCCTG } \\
\text { CTGCTCGGG }\end{array}$ \\
\hline & $C(593) T$ & $\begin{array}{l}\text { TCCTCAGCGCAGTCGAAAGACTGAGGTGAGCCTCGGCCTGCGCTGCGGGACCCCGGAGCCTGAGCGGCCTGGCTATGGACC } \\
\text { TGTGGGGACCCGGCACAGGGCGGCCCTGCTGCTCGGGTC[C/n]CTAGCCAGATGCGTCTTGGTCATCCAGCTCAGCTTCTCTTCT } \\
\text { CCGGGGATCTGGCGACATCCCTTCTTCAGCCAGAGGACACCACGGAGGAGCAGAGGACCCGCGCTCCAAGTCTCCAGGAT } \\
\text { GTGCCCAT }\end{array}$ \\
\hline exon 3 & $\begin{array}{l}\mathrm{G}(321607) \underline{A} \\
\mathrm{rs} 36596918\end{array}$ & $\begin{array}{l}\text { ACCACTGCTGCCCGGGGACTTCCCCAGGGCTATCTCAAACATGCACTTCCTGTCTCCAACAGGGCCAAGGTTAAGAAAGGAGT } \\
\text { GAGCATTGTTGGGGTGAGAGCCAGCAGCTCTTCAATCTGGGATGTCA[G/A] } \\
\text { ACAGAGCACTGAGTACACTGGGAAATATGCTCCG } \\
\text { GCCGTCATCGTTTGTCAGAAGAAATCTGCTGGCTCCGAAAAGAGGTAAGTCCCACCTGGCCTGTGCTGTGCTCAGACTCTCTG } \\
\text { CCATGGTGGCTTC }\end{array}$ \\
\hline \multirow[t]{3}{*}{ intron 3} & $\begin{array}{l}\Delta(360972-6) \\
\text { rs237178791 }\end{array}$ & $\begin{array}{l}\text { GTAACAAGGACTGGGAAAGATGTGGACAGACACTCACAAGAAGCTGATAGAATGTAATTTAGTCCAACTGCTGCCATGGGTT } \\
\text { TGGTATGTTGGGTTCTCAAAACAAACAAACAAACAAA[CAAA]ACTAACAAAACACCAAACCAAGACAACCCAAACAAAC } \\
\text { CATCATCATCAACAACAACAACAACAACA[ACA]GCCTTGAAATCAACTTGAGTATATGCATACTGTGTITCTGTAGGCA } \\
\text { TCTGAGAGAACAGAATTGCTAATCAGAAAAGCAGCAAA }\end{array}$ \\
\hline & $\begin{array}{l}C(322141) \underline{T} \\
\mathrm{rs} 13478520\end{array}$ & $\begin{array}{l}\text { TTGCCCAGCTGTGAATTGCCTGTTCTAATTTGGTGGCTATCAGAAGCAGATCCGGGTITAATTITCTCCCCGGGGTGATTCAC } \\
\text { GATCGCCTGTAATTCCACAGCGGG[C/[_]GGCCCACTGTTCCATCAGAAAGTGCCCCCTGCACAAATA } \\
\text { CAATCAAATGCAGTATGTTGACATCAGCAGCGCCGTAAAGGTGGGCTGCACCCTGCCTGTAGAAGAGCGAG }\end{array}$ \\
\hline & $\begin{array}{l}\mathrm{A}(361209) \mathrm{T} \\
\mathrm{rs} 6387514\end{array}$ & $\begin{array}{l}\text { AACAGAATTGCTAATCAGAAAAGCAGCAAAACATGATTATGAGACGTGATAGGCCCATGTGCTATCATGTGTGGAGTGTGCAGG } \\
\text { CTITAAGCATGCAGACATTCCTACCATCGGCGCCTG[A/I]AATCCTAAGTGGTGGGTGTATAACTAGATGCTCTCTGCGCAGAGT } \\
\text { TTAGCAGTCTTATGTTCCCACACTCTCCAGACCAGATTCTाTाTTGATGTCCCTTACTCTGTACAGAAAAATCCCCCTCTTGT }\end{array}$ \\
\hline intron 5 & $\mathrm{~T}(594085) \underline{A}$ & $\begin{array}{l}\text { TGACGAAGACGTGGTTAAGGCAAGTGGATGGTTCCTCGCCCATTAGGAAGCCCATAGTTACACGGGCATGGGTGTTTATC } \\
\text { CCCAGCTGAGATCTGTGGGGTCTGCAGGGTCCTCCCATTCTGGTTTCTT/A]GCAATTAAATCAGGAGTGTC } \\
\text { TCCCACCCCAACGCCAAATAGGATATACACTGCTATAACCCATGCCTGGGCTGTGAGACATTCTTCTGTGCCT }\end{array}$ \\
\hline exon 9 & $\begin{array}{l}\mathrm{A}(673963) \mathrm{G} \\
\mathrm{rs} 13478518\end{array}$ & $\begin{array}{l}\text { AATCTTGCCAGAAGTCTAAGCGGAAGAGCGTGCTGGCTGTGGGAACAGCCAGCATCAAGGTTAAATTTGGACAGAATGATGC } \\
\text { AAACCCCAACAGCAGTGAGAGTGGACACCTTGGGGCTGGGCTTCATGT[A/G]GAGAATATCAATGACAGGAGGTCC } \\
\text { AAAAAGCCTITCAGGAATGGGGGAGCCCCGAGGGGCCATTCTACAGCAGCTCATCCATGGGGCTCATGGAGG } \\
\text { GATGGGGCAGTACCACCAAGAGGCCAACTTC }\end{array}$ \\
\hline
\end{tabular}

Fig. 3g). AAV-eGFP injected into aCC could confirm viral spread (Fig. 3h).

Bidirectional nature of anxiety phenotype and plasticity of the Tmem132d gene

One-way ANOVA revealed overall statistically significant difference in both percent time spent on open arm of EPM $\left(F_{3,24}=117.3 ; p<0.001\right.$ : Fig. $\left.4 a\right)$ and light compartment of $\operatorname{LDB}\left(F_{3,24}=31.72 ; p<0.001\right.$ : Fig. $\left.4 \mathrm{c}\right)$, respectively. Furthermore, Bonferroni's post hoc test uncovered that HAB-EE spent significantly higher percent time on open arm of EPM and light compartment of LDB in comparison to $\mathrm{HAB}-\mathrm{SH}$ (Fig. 4a, c, respectively). While, LAB-UCMS spent significantly lower percent time on 
Table 8 Primers used for bisulfite sequencing of the Tmem132d gene in HAB vs. LAB mice

\begin{tabular}{lll}
\hline Primer & Orientation & Primer sequence $\left(\mathbf{5}^{\prime} \rightarrow \mathbf{3}^{\prime}\right)$ \\
\hline M13 tailed Bisulfite sequencing & & \\
Tmem132d & Forward & TGTAAAACGACGGCCAGTGGAGTGATGTTGGGTTITITT \\
Tmem132d & Reverse & CAGGAAACAGCTATGACCTTTAAACCCCACCCTTCTAAA \\
M13 tailed genomic DNA sequencing & & \\
Tmem132d & Forward & TGTAAAACGACGGCCAGTGGAGTGATGCTGGGTTCCTCT \\
Tmem132d & Reverse & CAGGAAACAGCTATGACCTTTAAGCCCCACCCTTCTGGA \\
\hline
\end{tabular}

Table 9 Primers used for in vitro methylation studies

\begin{tabular}{lll}
\hline Primer & Orientation & Primer sequence $\left(\mathbf{5}^{\prime} \boldsymbol{\rightarrow} \mathbf{3}^{\prime}\right)$ \\
\hline LAB Tmem132d & Forward & GTATTATGTATCTTGGTGTGAGTT[5MedC]GCCTTAGATACCCTGGAAGGCATG \\
LAB Tmem132d & Reverse & CATGCCTTCCAGGGTATCTAAGG[5MedC]GAACTCACACCAAGATACATAATAC \\
HAB Tmem132d & Forward & GTATTATGTATCTTGGTGTGATT[5MedC]GCCTTGGATACCCTGGAAGGCATG \\
HAB Tmem132d & Reverse & CATGCCTTCCAGGGTATCCAAGG[5MedC]GAACTCACACCAAGATACATAATAC \\
\hline
\end{tabular}

open arm of EPM and light compartment of LDB (Fig. 4a, c, respectively). Moreover, there was no difference in total distance traveled in both EPM and LDB test amongst the four groups (Fig. 4b, d, respectively).

One-way ANOVA also revealed an overall significant difference $\left(F_{3,21}=12.69 ; p<0.001\right.$; Fig. 4 e $)$ in Tmem132d gene expression in aCC with Bonferroni's post hoc test uncovering significantly enhanced expression in HAB-EE compared to HAB-SH $(p<0.001)$. Moreover, unpaired $t$ test revealed a trend towards significance with higher gene expression in HAB-SH compared to LAB-SH $\left(t_{11}=2.047\right.$; $p=0.0653$ ), which validates earlier studies ${ }^{15}$. We also observed a trend towards significantly decreased gene expression in LAB-UCMS compared to LAB-SH $\left(t_{10}=\right.$ $1.953 ; p=0.0794)$.

\section{ChIP to assess in vivo POLR2A binding}

One-way ANOVA with Bonferroni's correction of ChIP data confirmed a significantly higher binding of POLR2A at the HAB-specific guanine nucleotide at $(-310)$ locus compared to the corresponding LAB-specific adenine nucleotide $(p<0.01 ;$ Fig. 4f). In addition, we found enhanced binding of POLR2A to the HAB-EE $(-310)$ locus compared to respective HAB-SH region $(p<0.01)$. Student's $t$ test revealed lower POLR2A binding in the LAB-UCMS (-310) locus in comparison to corresponding LAB-SH locus $(p<0.001)$. In a separate set of immunoprecipitated DNA from mouse brain tissue, higher occupancy of POLR2A was detected in the promoter region of the housekeeping gene beta-actin and sparse POLR2A binding in a genetic desert on chromosome 6 . Thus, both positive and negative control primers (Active motif) confirmed their role as transcriptionally active and inactive chromatin marks, respectively.

\section{TMEM132D methylation is associated with Positive Life} Events and rs7309727 SNP variants in anxiety disorder patients

In previous studies, rs7309727 was found to be associated with PD with higher risk for $\mathrm{T}$ allele carriers ${ }^{16}$. Two CpGs (cg26322591 and cg03283235) located in the TMEM132D gene were significantly hypermethylated $\left(P_{\mathrm{FDR}}=0.0296\right)$ in $\mathrm{T}$ homozygotes anxiety patients experiencing a higher number of positive life events while CT heterozygotes showed lower methylation levels with a higher number of positive life events experienced. We also performed the analysis for association with negative life events and did not find any significant results (Fig. 5a, b).

\section{POLR2A enrichment across TMEM132D gene body}

There was substantial enrichment of POLR2A across TMEM132D gene, including the intronic regions in $\mathrm{K} 562$ and GM78 cell lines (Fig. 5c).

\section{Discussion}

Selective breeding of mice over several generations causes genetic variation associated with the trait anxiety to be represented at a higher frequency, leading to 


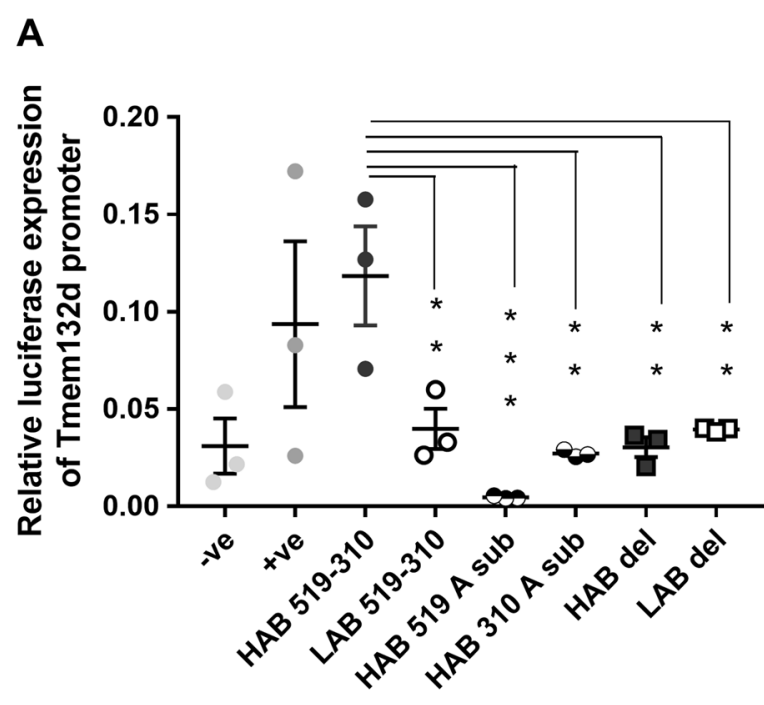

$\mathrm{n}=3$ for each group and

represent independent assays

performed in triplicate

B
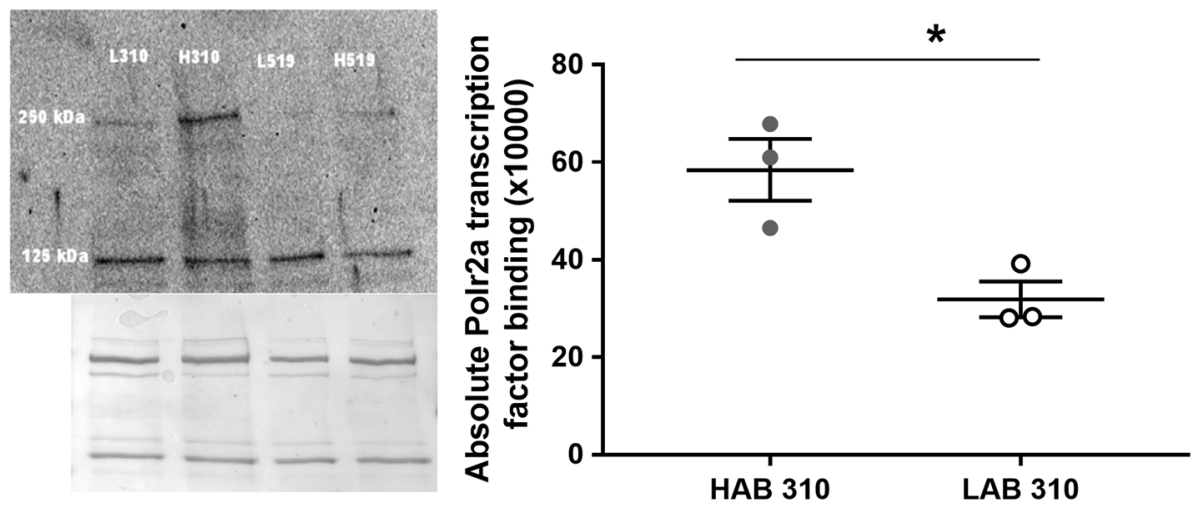

(3)

(3)

C

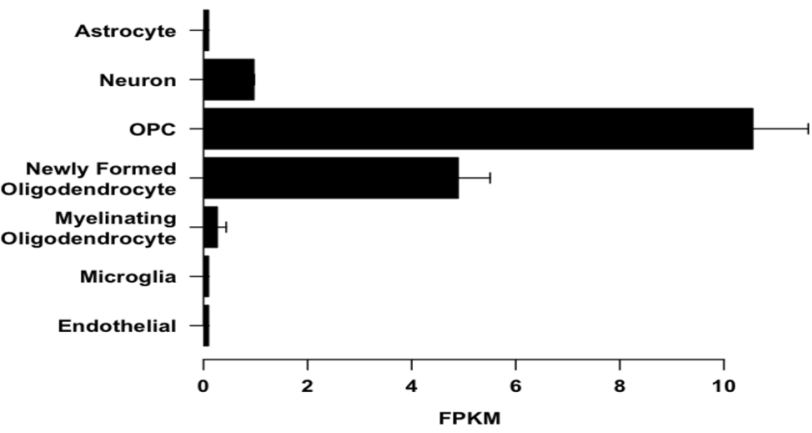

Fig. 2 a Dual luciferase assay with HAB vs. LAB Tmem132d promoter constructs. Data are shown as mean \pm SEM; ${ }^{* *} p<0.01$; ${ }^{* * *} p<0.001$; in comparison with HAB-specific SNPs $(-519) \mathrm{G}$ and $(-310) \mathrm{G}$. Firefly luciferase data were normalized to Gaussia activities and are presented as relative expression \pm SEM of three independent experiments performed in triplicate. Negative ( $-v e)$ and positive (+ve) controls represent pGL3 basic and SV40-pGL3 vectors, respectively. b Semi-quantitative western blots and representative image (upper) for POLR2A transcription factor binding to rs233264624 with the HAB-specific (-310)G vs. LAB-specific A(-310) along with corresponding colloidal silver staining (lower), which served as loading control. The data are shown as means \pm SEM, ${ }^{*} p<0.05$ ( $n=3$ for each group and represent independent assays performed in triplicate). $\mathbf{c}$ RNA- seq data showing Tmem 132d differential expression calculated as the Fragments Per Kilobase of transcript per Million mapped reads (FPKM) of a given cell type divided by the average FPKM of all other cell types (source: Zhang et al 2014) ${ }^{14}$ 
A

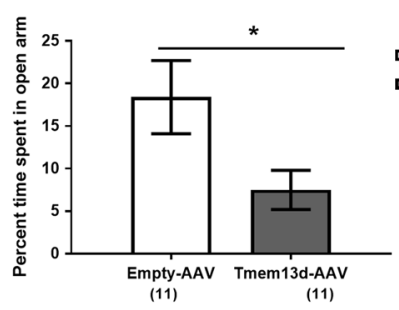

C

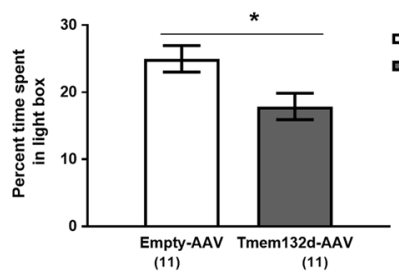

E

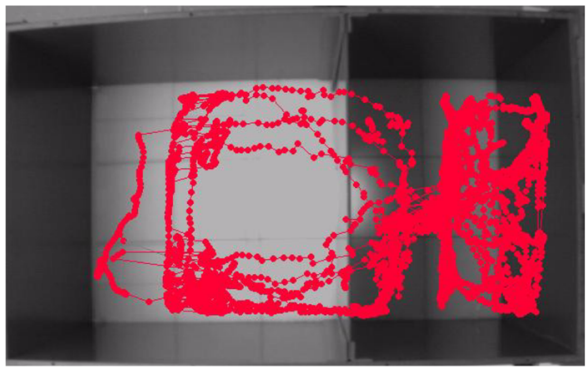

Empty-AAV

G

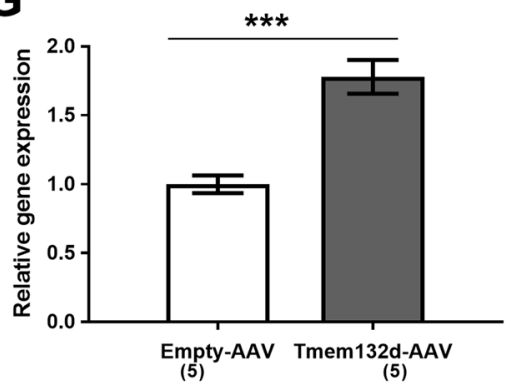

B

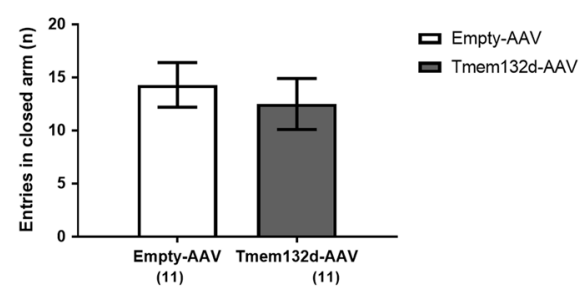

D

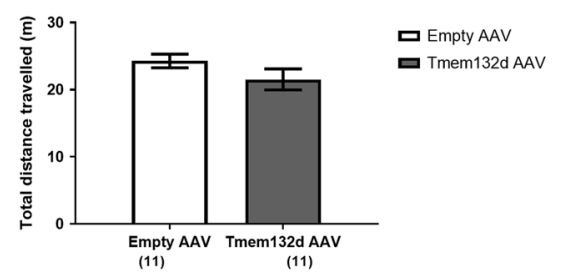

F

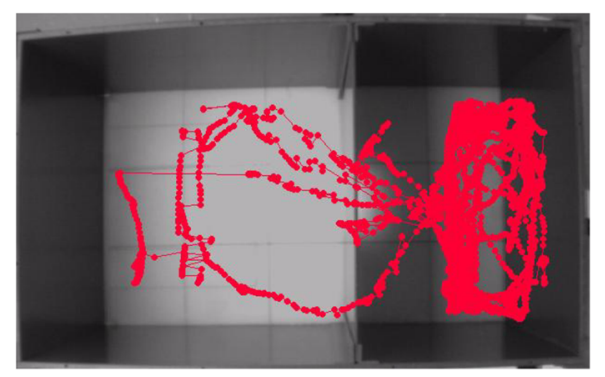

Tmem132d-AAV
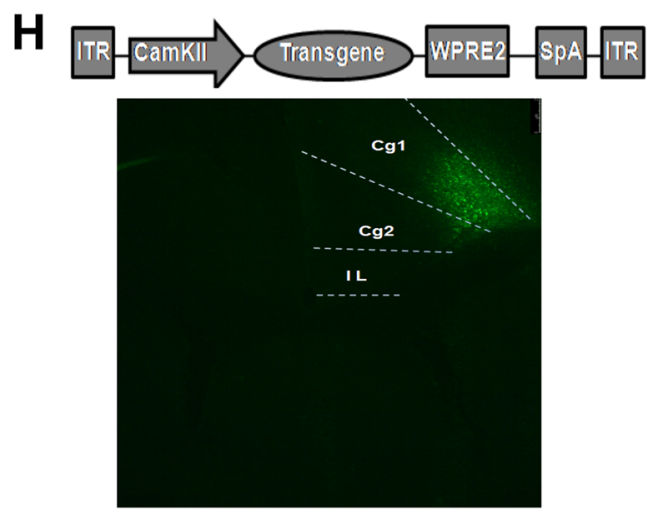

Fig. 3 Adeno-associated virus mediated overexpression of Tmem 132d or eGFP in the anterior cingulate cortex of C57BL/6 J mice. Percent time spent in open arm (a), and entries in closed arm (b) on the elevated-plus maze. Percent time spent in light compartment (c), and distance traveled (d) in the light-dark box test. Representative image of animal movement tracks of empty-AAV (e) vs. Tmem132d-AAV (f) injected C57BL/6 J mice. (g) Validation of Tmem132d mRNA expression in the anterior cingulate cortex of empty-AAV vs. Tmem132d-AAV injected C57BL/6 J mice. The data are shown as means \pm SEM, numbers in parentheses indicate the group size; ${ }^{*} p<0.05$, ${ }^{* * *} p<0.001$. (h) Representative image showing enhanced green fluorescent protein expression in anterior cingulate cortex (Cg1, Cg2) of C57BL6/J mice 


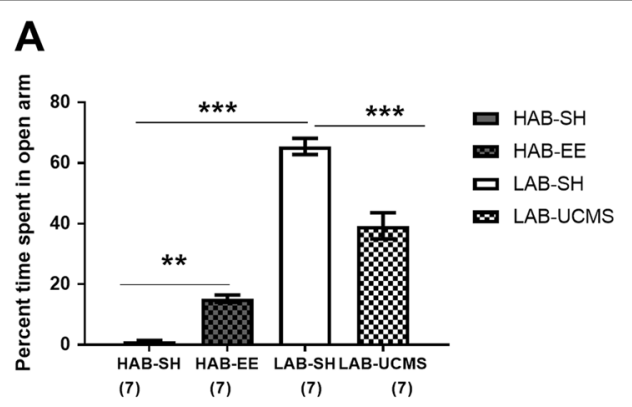

B
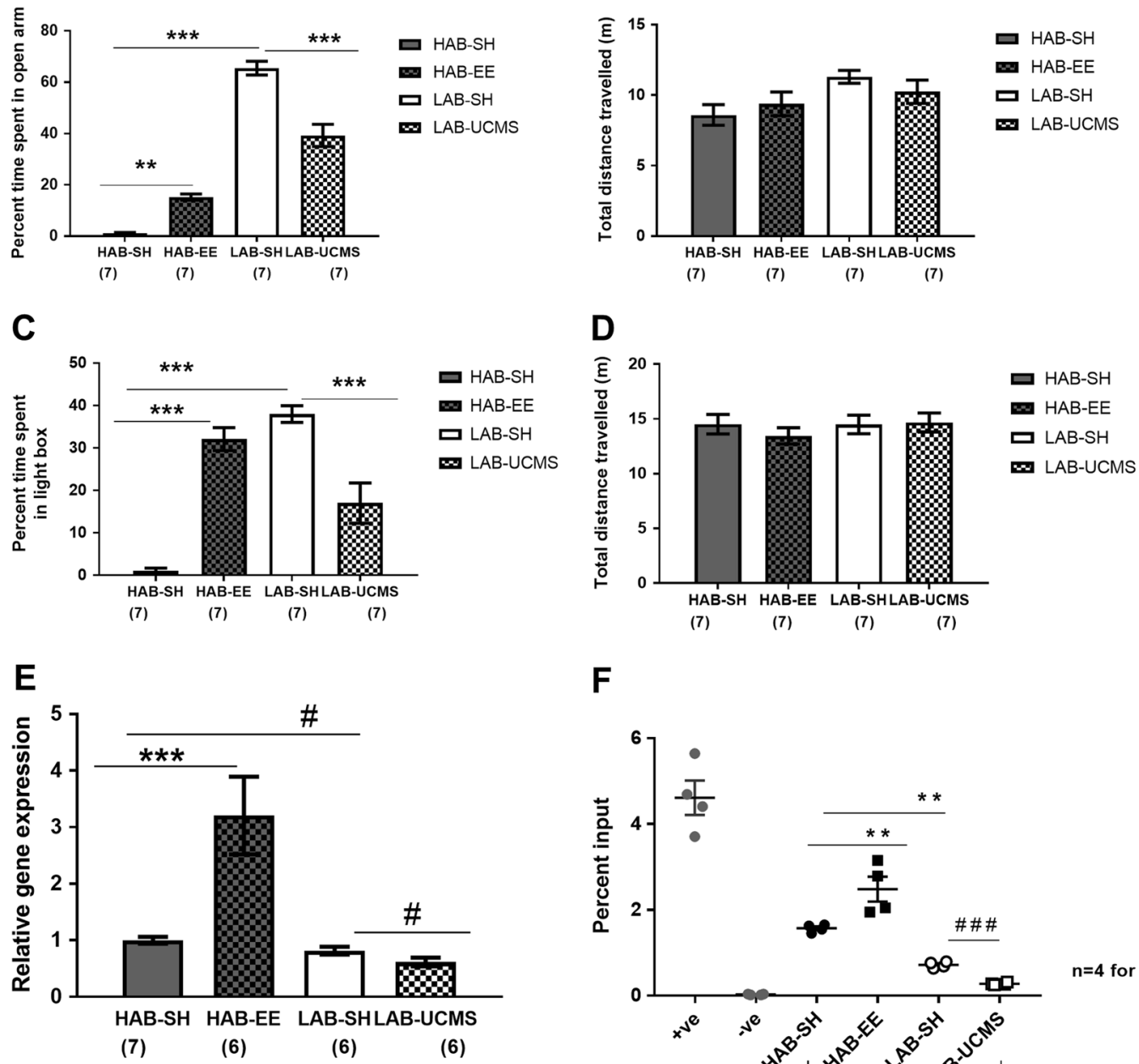

$\mathbf{F}$

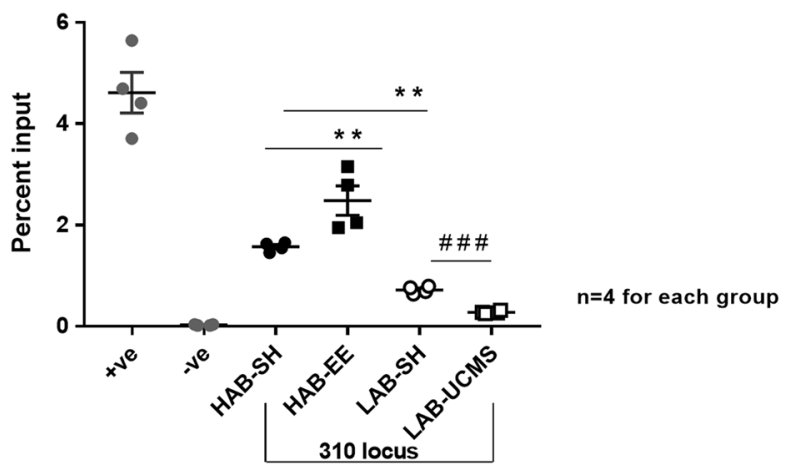

Fig. 4 Effect of environmental enrichment (EE) and unpredictable chronic mild stress (UCMS) on HAB and LAB mice, respectively. Percent time spent in open $\operatorname{arm}(\mathbf{a})$, and total distance traveled (b) on the elevated-plus maze. Percent time spent in light compartment (c), and total distance traveled (d) in the light-dark box test. e Relative Tmem 132d mRNA expression in HAB-SH, HAB-EE, LAB-SH and LAB-UCMS in the anterior cingulate cortex. Data are shown as means \pm SEM, numbers in parentheses indicate the group size; ${ }^{*} p>0.05,{ }^{* *} p<0.01,{ }^{* * *} p<0.001$. f Chromatin immunoprecipitation assay for POLR2A binding to (rs233264624) A(-310)G locus of HAB-SH/ HAB-EE/ LAB-SH/ and LAB-UCMS. Negative (-ve) and positive (+ve) controls represent occupancy of POLR2A at the active and inactive chromatin marks. The data are shown as means \pm SEM with $n=4$ for each group. ${ }^{* *} p<$ $0.01,{ }^{\# \# \# p} p<0.001$ (t test)

homozygosity at loci conferring this trait ${ }^{48}$. Anxiety disorders are spread across a continuum in a form of normal distribution with HAB and LAB denoting the two opposite extreme ends. The TMEM132D gene was first identified through genome wide association study in PD patients, in which intronic SNPs were associated with anticipatory anxiety in these patients as well as to severity of anxiety symptoms in patients with major depression or panic attacks, but not to the severity of depressive symptoms ${ }^{15}$. This suggests that the genetic variants are specific for the severity of experienced anxiety but not restricted to any particular disorder ${ }^{15}$. One of the associated variants showed regulatory function associating with higher Tmem132d mRNA expression in the frontal cortex of postmortem brain tissue, suggesting their functional role in anxiety pathophysiology ${ }^{15}$. Notably, a recently published study investigating quantitative trait loci in fear conditioning in mice revealed Tmem132d as suggestive locus for fear acquisition supporting its involvement in anxiety circuits ${ }^{20}$. Concurrently, in the $\mathrm{HAB} /$ LAB mouse model, Tmem132d was identified through microarray analysis and later confirmed independently through $\mathrm{qPCR}$ to be higher expressed in the aCC of HAB-SH compared to LAB-SH mice and intermediate 
A

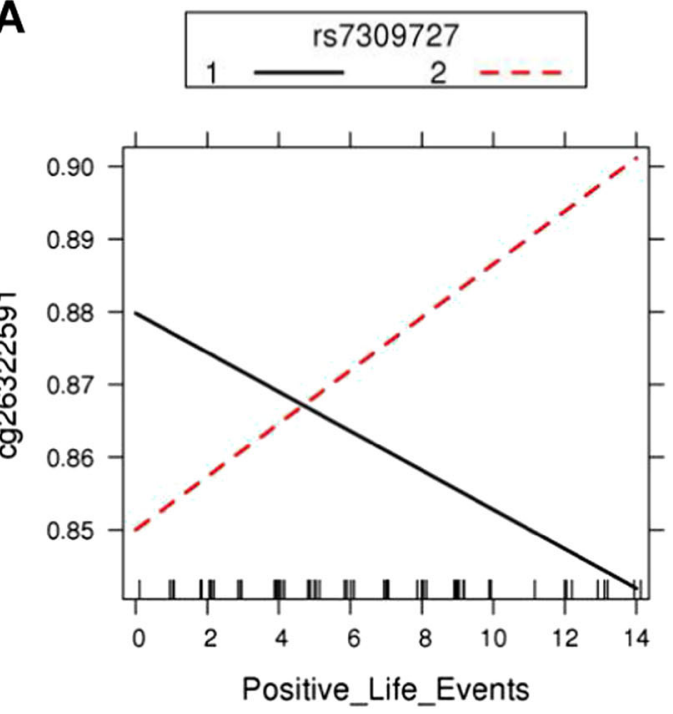

B
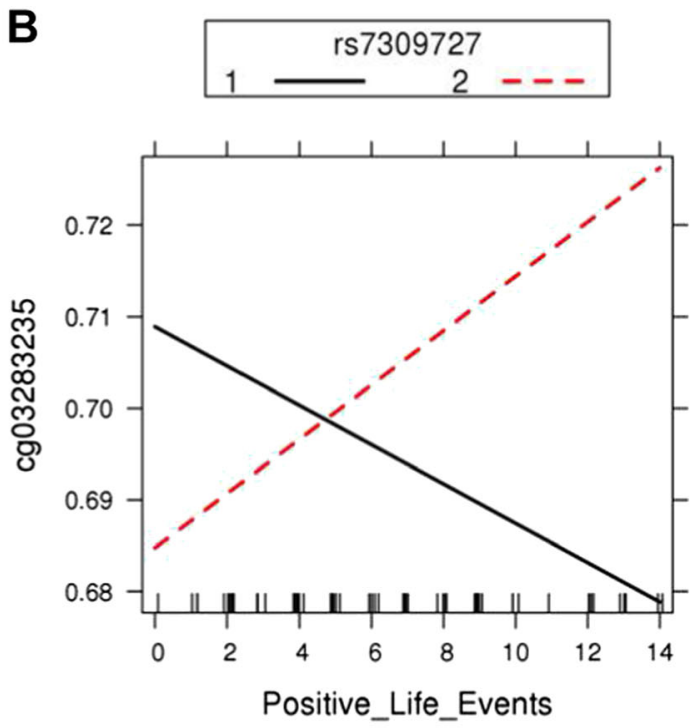

C

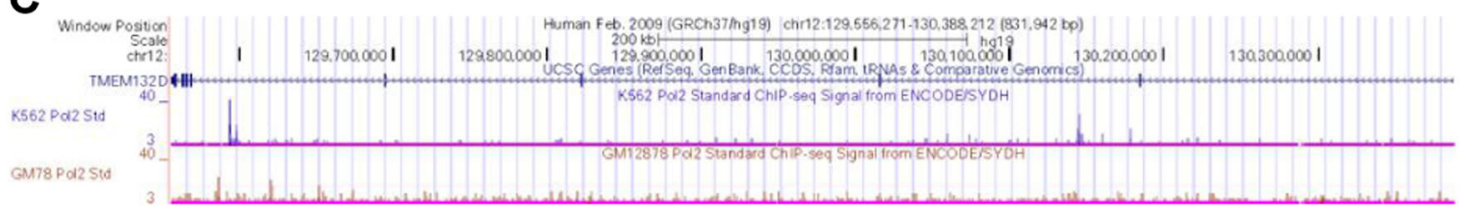

Fig. 5 Interaction plot of (a) cg26322591 and (b) cg03283235 with Positive Life Events depending on rs7309727 genotypes $(1=C T, 2=T$ ). $X$-axis: methylation levels expressed as $\beta$-values. Y-axis: Number of positive life events experienced. (c) Illustration of the RNA polymerase II ChIP-seq data from ENCODE project for K562 and GM78 human cell line showing POLR2A enrichment across TMEM132D gene body in UCSC genome browser on Human Feb. 2009 (GRCh37/hg19) Assembly

levels in CD-1 mice ${ }^{15}$. There was no such differential expression observed in the central, basolateral amygdala, hypothalamic paraventricular nucleus, and dentate gyrus of $\mathrm{HAB} / \mathrm{LAB}$ mice $^{15}$.

To study the genetic underpinnings behind this differential expression, we sequenced Tmem132d and found two SNPs $A(-310) G$ and $A(-519) G$ in the putative promoter of the gene. We observed two guanine $(G)$ residues in the HAB Tmem132d promoter in place of the adenine (A) residues in the corresponding $\mathrm{LAB}$ locus. We also observed a SNP in the 3rd exon (rs36596918) that causes substitution from arginine to lysine, however, the functional significance of this SNP is currently unknown. Also, another SNP in the 9th exon (rs13478518), part of untranslated region, was found to co-segregate with anxiety-related behavior in an F2 panel, independent of depression-like behavior and locomotor activity ${ }^{15}$, advocating a causal role of the Tmem132d gene in anxietyrelated behavior.

The rs36596918 was utilized as allele-specific tag to quantify the $\mathrm{HAB}$ and $\mathrm{LAB}$ allele-specific contribution to Tmem132d expression in cross-mated (HABxLAB) F1 offspring and CD-1 mice and were found to have higher
HAB allele-specific expression in comparison to LAB allele-specific. Similar data were observed with F1 offspring and CD-1 mice using rs13478518 as allele-specific tag (data not shown). To dissect the functional importance of promoter SNPs, we amplified and cloned the HAB/LAB Tmem132d promoters in gene reporter (luciferase) vectors. The resultant assay revealed higher luciferase expression, suggesting that the two SNPs indeed caused higher Tmem132d mRNA expression. Furthermore, to understand the putative transcription factor that might modulate this differential expression, we carried out in silico analysis, which suggested binding of nuclear factor I/C (NFI/C; CCAAT-box binding transcription factor) at the HAB -specific locus (-519)G and general transcription factor II B (GTFIIB) at the proximal (-310) G HAB position, respectively. Overexpression or short hairpin RNA (shRNA) knockdown of NFI/C or GTFIIB did not alter luciferase expression of the $\mathrm{HAB} / \mathrm{LAB}$ Tmem132d promoter (data not shown).

Nonetheless, NFI/C has been shown to be involved in eukaryotic transcription $^{49}$ and colocalizes with RNA polymerase $\mathrm{II}^{50}$. Moreover, studies have shown that binding of $\mathrm{NFI} / \mathrm{C}$ is blocked with $\mathrm{CpG}$ methylation 
resulting in increased promoter activity of Igf2 $2^{51}$. However, bisulfite sequencing of standard-housed $\mathrm{HAB}$ and LAB Tmem132d promoter harboring a CpG island did not reveal any difference in total percentage of DNA methylation (Supplementary Data S2). Moreover, selective or complete methylation of HAB/LAB Tmem132d promoter region did not alter luciferase expression i.e., promoter activity (data not shown). But it is plausible that DNA methylation might bind at other regulatory regions in the gene body and regulate its expression. It is worth noting that the GTFIIB that binds -310 SNP is involved in the formation of the RNA polymerase II preinitiation complex and aids in initiation of transcription $^{52,53}$. Thus, both the guanine residue in HAB Tmem132d promoter seem to interact with RNA polymerase II, and therefore, we carried out oligonucleotide pull-down assay for one of its largest subunit i.e., RNA Polymerase II subunit B1 (POLR2A). We observed significantly higher binding of POLR2A to the HAB-specific $(-310) \mathrm{G}$ position compared to corresponding LAB locus, suggesting that the HAB-specific $(-310) G$ variant causes a gain-of-function leading to higher binding of POLR2A and possibly, increase in transcription. Thus, we could first identify one regulatory component in the Tmem132d gene, which is relevant for a behavioral phenotype in mice. We utilized four different commercial antibodies to examine TMEM132D protein expression difference in aCC of HAB-SH and LAB-SH along with samples from Tmem132d $d^{(-)}$knockout (KO) mice, however, none of them were specific for the target gene (Supplementary Data S3).

We also utilized AAV-mediated overexpression of Tmem132d in the aCC of C57BL/6J mice and observed lower percent time spent in open arm of EPM and light compartment of the LDB confirming its role in mediating an anxiogenic phenotype. The frequency of anxiety disorders have been studied in monozygotic and dizygotic twins, in which the contribution of genetic factors ranges from 30 to $40 \%$, while the rest can be attributed to differences in environment ${ }^{3}$. Thus, to study the gene-environmental interactions, we subjected $\mathrm{HAB}$ to $E E$ and $L A B$ were exposed to UCMS to shift their genetically predisposed anxiety phenotype towards normality.

HAB-EE mice were indeed found to have lower anxiety levels as indicated by higher percent time spent on open arm of EPM and light compartment of LDB in comparison to HAB-SH. While, LAB-UCMS mice had higher anxiety response compared to $\mathrm{LAB}-\mathrm{SH}$ as indicated by lower percent time spent on open arm of EPM and light compartment of LDB. Intriguingly, in the $\mathrm{aCC}$, we found that the HAB-EE had augmented Tmem132d mRNA expression compared to HAB-SH. On the other hand, LAB-UCMS had lower Tmem132d mRNA expression in comparison to LAB-SH. We also found significantly higher POLR2A occupancy at $(-310)$ G locus of HAB-EE compared to HAB-SH, suggesting increased transcription, which explain the observed higher mRNA expression differences. On the other hand, there was significantly lower POLR2A binding at (-310)A locus of LAB-UCMS in comparison to LAB-SH, further explaining their attenuated level of gene expression. Thus, there is a bidirectional modulation of Tmem132d mRNA expression in HAB-EE and LAB-UCMS compared to their respective SH mice.

Studies have proposed that hippocampal neurogenesis $^{54}$, synaptogenesis enhancement ${ }^{55}$, neuropeptideY ${ }^{56}$ and serotonin levels in frontal cortex ${ }^{57}$ may underlie EE induced decrease in anxiety. Thus, it is plausible that $\mathrm{EE}$ causes decrease in anxiety independent of Tmem132d in $\mathrm{HAB}$ mice. The exact regulatory function of TMEM132D gene is unknown.

From our previous studies, we predicted that an enhanced gene expression would lead to higher anxiety levels what holds true for the acute vector overexpression in mice. However, an early and prolonged positive environmental stimulation also seems to increase the gene expression levels in our animal model (HAB), whereas a prolonged mild stressful condition in LAB has opposite effects. This suggests time-dependent differential effects of the environment on Tmem132d gene regulation. Fig. 6 summarizes major behavioral and molecular alterations observed in our animal model.

In order to bring animal and human results together, we also looked for methylation difference in patients with PD in the TMEM132D gene region. The question was whether we can see any influence on methylation profile in dependence of life events in patients with risk alleles. We see two interesting associations, which show that patients homozygotic for the risk allele of rs7309727 show increased methylation in two CpGs with higher positive life events. In contrast, in patients having just one risk allele, we have an opposite effect. As increased methylation levels is mostly associated with lower gene expression. This is again in opposite to the findings in the HABEE mice. Further studies with analysis of control probands and different environmental exposure settings are needed to explain these effects. ENCODE project revealed POLR2A enrichment across TMEM132D gene body in K562 and GM78 cell lines, suggesting its importance in regulating TMEM132D gene expression ${ }^{43,44}$. RNA polymerase II pausing in proximal promoter region is widespread across genes involved in signal responsive pathways and has been described as a regulatory mechanism to diverse environmental cues in higher eukaryotes ${ }^{58,59}$. In summary, we see a modulatory function of environmental cues on Tmem132d gene expression in mice and methylation differences in risk allele 


\begin{tabular}{|c|c|c|c|c|}
\hline Groups & HAB-SH & HAB-EE & LAB-SH & LAB-UCMS \\
\hline \multicolumn{5}{|c|}{ Basal changes } \\
\hline Anxiety phenotype & +1+ & NA & ++ & NA \\
\hline Tmemr132d expression & +1+ & NA & ++ & NA \\
\hline SNPs & $\checkmark$ & NA & $\mathbf{x}$ & NA \\
\hline $\begin{array}{l}\text { Transcription factor: } \\
\text { Polr2a }\end{array}$ & ++4 & NA & ++ & NA \\
\hline $\begin{array}{l}\text { Promoter: } \\
\text { DNA methylation }\end{array}$ & - & NA & - & NA \\
\hline \multicolumn{5}{|c|}{ Post-emironmental manipulations (Le EE for HAB and UCuS for LAB, respectively) } \\
\hline Anxiety phenotype & +++1 & + & ++ & +++ \\
\hline Tmemr132d expression & +++1 & +1+1+ & ++ & + \\
\hline $\begin{array}{l}\text { Transcription factor. } \\
\text { Pour2a }\end{array}$ & H+1+ & +1+H+ & ++ & + \\
\hline
\end{tabular}

Fig. 6 Summary of major behavioral and molecular events of HAB-SH/HAB-EE/LAB-SH/LAB-UCMS. Plus(+) sign(s) indicate increase in strength of a particular behavioral or molecular parameter. Minus (-) sign stands for no difference. NA stands for not applicable

patients with $\mathrm{PD}$ in response to positive life events, however, the direction of the changes remains unclear.
Received: 5 October 2016 Revised: 30 July 2017 Accepted: 29 August 2017 Published online: 20 December 2017

\section{Acknowledgements}

We thank Dr. Theo Rein and Prof. Inga Neumann for the vectors and permission to carry out AAV experiment, respectively. We also thank Prof. Tomoko Yonezawa and Dr. Jan Deussing for the Tmem132d cDNA plasmid and Tmem132d(-) KO brain tissue, respectively. We would also thank Marina Zimbelmann, Ruth Jelinek, Rodrigue Maloumby, Markus Nußbaumer, Anne Stündl and Dr. Stephan Guggenhuber for their excellent technical assistance and initial guidance with design of AAV plasmid, respectively. We also thank Prof. Michael Rehli at Universität Klinikum Regensburg for the CpG free vector and Prof. Li-Huei Tsai at Massachusetts Institute of Technology, USA for the CaMKII-H1 promoter-empty-mcherry-AAV vector. The investigations of the MPIP cohort were supported by ERA-NET NEURON (AnxBio).

\section{Author details}

${ }^{1}$ Max Planck Institute of Psychiatry, 80804 Munich, Germany. ${ }^{2}$ Department of Behavioral and Molecular Neurobiology, University of Regensburg, Regensburg, Germany. ${ }^{3}$ Department of Normal Physiology, Sechenov First Moscow State Medical University, Moscow, Russia. ${ }^{4}$ Institute of Physiological Chemistry, University Medical Center of the Johannes Gutenberg University, Mainz, Germany. ${ }^{5}$ Lee Kong Chian School of Medicine, Nanyang Technological University, Singapore 308232, Singapore. 'Labor Becker und, 81671 Munich, Germany

\section{Competing interests}

The authors declare that they have no competing financial interest.

Publisher's note: Springer Nature remains neutral with regard to jurisdictional claims in published maps and institutional affiliations.

\section{Supplementary information}

The online version of this article (https://doi.org/10.1038/s41398-017-0025-2) contains supplementary material.

\section{References}

1. Gross, C. \& Hen, R. The developmental origins of anxiety. Nat. Rev Neurosci. 5, 545-552 (2004).

2. Kessler, R. C. \& Wang, P. S. The descriptive epidemiology of commonly occurring mental disorders in the United States. Annu. Rev. Public. Health. 29, 115-129 (2008).

3. Hettema, J. M., Prescott, C. A. \& Kendler, K. S. A population-based twin study of generalized anxiety disorder in men and women. J. Nerv. Ment. Dis. 189, 413-420 (2001).

4. Hettema, J. M., Prescott, C. A., Myers, J. M., Neale, M. C. \& Kendler, K. S. The structure of genetic and environmental risk factors for anxiety disorders in men and women. Arch. Gen. Psychiatry. 62, 182-189 (2005).

5. Rosenzweig, M. R., Bennett, E. L., Hebert, M. \& Morimoto, H. Social grouping cannot account for cerebral effects of enriched environments. Brain. Res. 153, 563-576 (1978).

6. van Praag, H., Kempermann, G. \& Gage, F. H. Neural consequences of environmental enrichment. Nat. Rev. Neurosci. 1, 191-198 (2000).

7. Sztainberg, Y., Kuperman, Y., Justice, N. \& Chen, A. An anxiolytic role for CRF receptor type 1 in the globus pallidus. J. Neurosci 31, 17416-17424 (2011).

8. Sotnikov, S. V. et al. Bidirectional rescue of extreme genetic predispositions to anxiety: impact of $\mathrm{CRH}$ receptor 1 as epigenetic plasticity gene in the amygdala. Transl. Psychiatry. 4, e359 (2014).

9. Mineur, Y. S., Belzung, C. \& Crusio, W. E. Effects of unpredictable chronic mild stress on anxiety and depression-like behavior in mice. Behav. Brain. Res. 175 , 43-50 (2006).

10. Ohman, A. The role of the amygdala in human fear: automatic detection of threat. Psychoneuroendocrinology 30, 953-958 (2005).

11. Hasler, G. et al. Cerebral blood flow in immediate and sustained anxiety. J. Neurosci. 27, 6313-6319 (2007).

12. Holzschneider, K. \& Mulert, C. Neuroimaging in anxiety disorders. Dialogues. Clin. Neurosci. 13, 453-461 (2011).

13. Nomoto, $\mathrm{H}$. et al. Molecular cloning of a novel transmembrane protein MOLT expressed by mature oligodendrocytes. J. Biochem. 134, 231-238 (2003). 
14. Zhang, Y. et al. An RNA-sequencing transcriptome and splicing database of glia, neurons, and vascular cells of the cerebral cortex. J. Neurosci. 34, 11929-11947 (2014).

15. Erhardt, A. et al. TMEM132D, a new candidate for anxiety phenotypes: evidence from human and mouse studies. Mol. Psychiatry. 16, 647-663 (2011).

16. Erhardt, A. et al. Replication and meta-analysis of TMEM132D gene variants in panic disorder. Transl. Psychiatry 2, e156 (2012).

17. Inoue, A. et al. Association of TMEM132D, COMT, and GABRA6 genotypes with cingulate, frontal cortex and hippocampal emotional processing in panic and major depressive disorder. Int. J. Psychiatry Clin. Pract. 19, 192-200 (2015).

18. Quast, C. et al. Rare variants in TMEM132D in a case-control sample for panic disorder. Am. J. Med. Genet. B. Neuropsychiatr. Genet. 159B, 896-907 (2012).

19. Haaker, J. et al. Higher anxiety and larger amygdala volumes in carriers of a TMEM132D risk variant for panic disorder. Transl. Psychiatry 4, e357 (2014).

20. Knoll, A. T., Halladay, L. R., Holmes, A. J. \& Levitt, P. Quantitative Trait Loci and a Novel Genetic Candidate for Fear Learning. J. Neurosci. 36, 6258-6268 (2016).

21. Bunck, M. et al. A hypomorphic vasopressin allele prevents anxiety-related behavior. PLOS ONE 4, e5129 (2009).

22. Franklin K. B. J., Paxinos G. The Mouse Brain In Stereotaxic Coordinates, 3rd ed. San Diego: Academic. 2007.

23. Czibere, L. et al. Profiling trait anxiety: transcriptome analysis reveals cathepsin $\mathrm{B}$ (Ctsb) as a novel candidate gene for emotionality in mice. PLOS ONE 6 e23604 (2011)

24. Gonik, M. et al. The endocrine stress response is linked to one specific locus on chromosome 3 in a mouse model based on extremes in trait anxiety. BMC Genomics 13, 579 (2012).

25. Farre, D. et al. Identification of patterns in biological sequences at the ALGGEN server: PROMO and MALGEN. Nucleic Acids Res. 31, 3651-3653 (2003).

26. Messeguer, $X$. et al. PROMO: detection of known transcription regulator elements using species-tailored searches. Bioinformatics 18, 333-334 (2002).

27. Schulke, J. P. et al. Differential impact of tetratricopeptide repeat proteins on the steroid hormone receptors. PLOS ONE 5, e11717 (2010).

28. Harper S., Speicher D. W. Detection of proteins on blot membranes. Curr. Protoc. Protein Sci. 10.8, 10.8.1-10.8.7 (2001).

29. Dittgen $T$ et al. Lentivirus-based genetic manipulations of cortical neurons and their optical and electrophysiological monitoring in vivo. Proceedings of the National Academy of Sciences of the United States of America 101, 18206-18211 (2004).

30. Guggenhuber, S., Monory, K., Lutz, B. \& Klugmann, M. AAV vector-mediated overexpression of CB1 cannabinoid receptor in pyramidal neurons of the hippocampus protects against seizure-induced excitoxicity. PLOS ONE $\mathbf{5}$, e15707 (2010)

31. Li, S., Tian, X., Hartley, D. M. \& Feig, L. A. The environment versus genetics in controlling the contribution of MAP kinases to synaptic plasticity. Curr. Biol. 16 2303-2313 (2006)

32. Arai, J. A., Li, S., Hartley, D. M. \& Feig, L. A. Transgenerational rescue of a genetic defect in long-term potentiation and memory formation by juvenile enrichment. J. Neurosci. 29, 1496-1502 (2009).

33. Willner, P., Towell, A., Sampson, D., Sophokleous, S. \& Muscat, R. Reduction of sucrose preference by chronic unpredictable mild stress, and its restoration by a tricyclic antidepressant. Psychopharmacology. 93, 358-364 (1987).

34. Livak, K. J. \& Schmittgen, T. D. Analysis of relative gene expression data using real-time quantitative $P C R$ and the 2(-Delta Delta $C(T))$ method. Methods $\mathbf{2 5}$ 402-408 (2001)

35. Slattery, D. A. et al. Selective breeding for high anxiety introduces a synonymous SNP that increases neuropeptide $\mathrm{S}$ receptor activity. J. Neurosci. 35 4599-4613 (2015)
36. Maier, W., Buller, R., Philipp, M. \& Heuser, I. The Hamilton Anxiety Scale: reliability, validity and sensitivity to change in anxiety and depressive disorders. J. Affect. Disord. 14, 61-68 (1988).

37. Bandelow, B. Assessing the efficacy of treatments for panic disorder and agoraphobia. II. The Panic and Agoraphobia Scale. Int. Clin. Psychopharmacol. 10, 73-81 (1995).

38. Ising, M., Lauer, C. J., Holsboer, F. \& Modell, S. The Munich vulnerability study on affective disorders: premorbid psychometric profile of affected individuals. Acta. Psychiatr. Scand. 109, 332-338 (2004).

39. Holmes, T. H. \& Rahe, R. H. The Social Readjustment Rating Scale. J. Psychosom Res. 11, 213-218 (1967).

40. Chen, Y. A. et al. Discovery of cross-reactive probes and polymorphic CpGs in the Illumina Infinium HumanMethylation450 microarray. Epigenetics 8, 203-209 (2013).

41. Fortin, J. P. et al. Functional normalization of 450k methylation array data improves replication in large cancer studies. Genome Biol. 15, 503 (2014)

42. Houseman, E. A. et al. DNA methylation arrays as surrogate measures of cell mixture distribution. BMC Bioinformatics 13, 86 (2012).

43. Raha, D. et al. Close association of RNA polymerase II and many transcription factors with Pol III genes. Proc. Natl Acad. Sci. USA 107, 3639-3644 (2010).

44. Rozowsky, J. et al. AlleleSeq: analysis of allele-specific expression and binding in a network framework. Mol. Syst. Biol. 7, 522 (2011).

45. Consortium, E. P. An integrated encyclopedia of DNA elements in the human genome. Nature 489, 57-74 (2012).

46. Kent, W. J. et al. The human genome browser at UCSC. Genome Res. 12 996-1006 (2002).

47. Rosenbloom, K. R. et al. The UCSC Genome Browser database: 2015 update. Nucleic Acids Res. 43, D670-D681 (2015). Database issue.

48. Falconer DS, Mackay TFC. An Introduction to Quantitative Genetics, 4th ed. Prentice Hall, London, 1996

49. Santoro, C., Mermod, N., Andrews, P. C. \& Tjian, R. A family of human CCAATbox-binding proteins active in transcription and DNA replication: cloning and expression of multiple cDNAs. Nature 334, 218-224 (1988).

50. Zhao, L. H. et al. BAF complex is closely related to and interacts with NF1/CTF and RNA polymerase $\|$ in gene transcriptional activation. Acta. Biochim. Biophys. Sin. 37, 440-446 (2005)

51. Jaenisch, R. \& Bird, A. Epigenetic regulation of gene expression: how the genome integrates intrinsic and environmental signals. Nat. Genet. 33 245-254 (2003). Suppl.

52. Kostrewa, D. et al. RNA polymerase II-TFIIB structure and mechanism of transcription initiation. Nature 462, 323-330 (2009).

53. Sainsbury, S., Niesser, J. \& Cramer, P. Structure and function of the initially transcribing RNA polymerase II-TFIB complex. Nature 493, 437-440 (2013).

54. Kempermann, G., Kuhn, H. G. \& Gage, F. H. More hippocampal neurons in adult mice living in an enriched environment. Nature 386, 493-495 (1997).

55. Bruel-Jungerman, E., Laroche, S. \& Rampon, C. New neurons in the dentate gyrus are involved in the expression of enhanced long-term memory following environmental enrichment. Eur. J. Neurosci. 21, 513-521 (2005).

56. Reichmann, F. et al. Environmental enrichment induces behavioural disturbances in neuropeptide Y knockout mice. Sci. Rep. 6, 28182 (2016).

57. Leger, $\mathbf{M}$. et al. Environmental enrichment duration differentially affects behavior and neuroplasticity in adult mice. Cereb. Cortex 25, 4048-4061 (2015).

58. Muse, G. W. et al. RNA polymerase is poised for activation across the genome. Nat. Genet. 39, 1507-1511 (2007)

59. Adelman, K. \& Lis, J. T. Promoter-proximal pausing of RNA polymerase ॥: emerging roles in metazoans. Nat. Rev. Genet. 13, 720-731 (2012). 